\title{
Algorithms and Applications for Universal Quantification in Relational Databases
}

\author{
Ralf Rantzau ${ }^{\mathrm{a}}$, Leonard D. Shapiro ${ }^{\mathrm{b}}$, Bernhard Mitschang ${ }^{\mathrm{a}}$ and Quan Wang ${ }^{\mathrm{c}}$ \\ a Computer Science Department, \\ University of Stuttgart, \\ Breitwiesenstr. 20-22, 70565 Stuttgart, Germany \\ b Computer Science Department, \\ Portland State University, \\ P.O. Box 751, Portland, OR 97201-0751, Oregon, USA \\ c Oracle Corporation, \\ Portland, Oregon, USA
}

Queries containing universal quantification are used in many applications, including business intelligence applications and in particular data mining. We present a comprehensive survey of the structure and performance of algorithms for universal quantification. We introduce a framework that results in a complete classification of input data for universal quantification. Then we go on to identify the most efficient algorithm for each such class. One of the input data classes has not been covered so far. For this class, we propose several new algorithms. Thus, for the first time, we are able to identify the optimal algorithm to use for any given input dataset.

These two classifications of optimal algorithms and input data are important for query optimization. They allow a query optimizer to make the best selection when optimizing at intermediate steps for the quantification problem.

In addition to the classification, we show the relationship between relational division and the set containment join and we illustrate the usefulness of employing universal quantifications by presenting a novel approach for frequent itemset discovery.

\section{Introduction}

Universal quantification is an important operation in the first order predicate calculus. This calculus provides existential and universal quantifiers, represented by $\exists$ and $\forall$, respectively. A universal quantifier that is applied to a variable $x$ of a formula $f$ specifies that the formula is true for all values of $x$. We say that $x$ is universally quantified in the formula $f$, and we write $\forall x: f(x)$ in calculus.

In relational databases, universal quantification is implemented by the division operator (represented by $\div$ ) of the relational algebra. The division operator is important for databases because it appears often in practice, particularly in business intelligence applications, including online an- alytic processing (OLAP) and data mining. In this paper, we will focus on the division operator exclusively.

Several algorithms have been proposed to implement relational division efficiently. These algorithms are presented in an isolated manner in the research literature-typically, no relationships are shown between them. Furthermore, each of these algorithms claims to be superior to others, but in fact each algorithm has optimal performance only for certain types of input data.

\subsection{The Division Operator}

To illustrate the division operator we will use a simple example throughout the paper, illustrated in Figure 1, representing data from a CS department at a university [8]. A course row repre- 
sents a course that has been offered by the department and an enrollment row indicates that a student has taken a particular course. The following query can be represented by the division operator:

"Which students have taken all courses offered by the department?"

As indicated in the table result, only Bob has taken all the courses. Bob is enrolled in another course (Graphics) but this does not affect the result. Both Alice and Chris are not enrolled in the Databases course. Therefore, they are not included in the result.

The division operator takes two tables for its input, the divisor and the dividend, and generates one table, the quotient. All the data elements in the divisor must appear in the dividend, paired with any element (such as Bob) that is to appear in the quotient.

In the example of Figure 1, the divisor and quotient have only one attribute each, but in general, they may have an arbitrary number of attributes. In any case, the set of attributes of the dividend is the disjoint union of the attributes of the divisor and the quotient. To simplify our exposition, we assume that the names of the dividend attributes are the same as the corresponding attribute names in the divisor and the quotient.

\subsection{Outline of the Paper}

The remainder of this paper is organized as follows. In Section 2, we present a classification of input data for algorithms that evaluate division within queries. Section 3 gives an overview of known and new algorithms to solve the universal quantification problem and classifies them according to two general approaches for division. In Section 4, we evaluate the algorithms according to both applicability and effectiveness for different kinds of input data, based on a performance analysis. In Section 5, we discuss the relationship between relational division and the set containment join. Section 6 illustrates a new approach to exploit division and set containment join to discover frequent itemsets. Section 7 gives an overview of related work. Section 8 concludes the paper and comments on future work.

\begin{tabular}{|c|c|c|}
\hline \multicolumn{2}{|c|}{ enrollment } & \multirow[b]{4}{*}{ course } \\
\hline student_id & course_id & \\
\hline Alice & Compilers & \\
\hline Alice & Theory & \\
\hline Bob & Compilers & course_id \\
\hline Bob & Databases & \multirow{2}{*}{$\begin{array}{l}\text { Compilers } \\
\text { Databases }\end{array}$} \\
\hline Bob & Graphics & \\
\hline Bob & Theory & \multirow{4}{*}{$\begin{array}{l}\text { Theory } \\
\text { (b) Divisor }\end{array}$} \\
\hline Chris & Compilers & \\
\hline Chris & Graphics & \\
\hline Chris & Theory & \\
\hline \multicolumn{3}{|c|}{ (a) Dividend } \\
\hline \multicolumn{3}{|c|}{ result } \\
\hline \multicolumn{2}{|r|}{ student_id } & \\
\hline \multicolumn{2}{|r|}{ Bob } & \\
\hline & (c) $\mathrm{Q}$ & \\
\hline
\end{tabular}

Figure 1 . enrollment $\div$ course $=$ result, representing the query "Which students have taken all courses?"

\section{Classification of Data}

This section presents an overview of the input data for division. We identify all possible classes of data based on whether it is grouped on certain attributes. For some of these classes, we will present efficient algorithms in Section 3 that exploit the specific data properties of a class.

\subsection{Input Data Characteristics}

The goal of this paper is to identify optimal algorithms for the division operator, for all possible inputs. Several papers compare new algorithms to previous algorithms and claim superiority for one or more algorithms, but they do not address the issue of which algorithms are optimal for which types of data $[3,4,8]$. In fact, the performance of any algorithm depends on the structure of its input data.

If we know about the structure of input data, we could employ an algorithm that exploits this structure, i.e., the algorithm does not have to restructure the input before it can start generating output data. Of course, there is no guarantee that such an algorithm is always "better" than 
an algorithm that requires previous restructuring. However, the division operator offers a variety of alternative algorithms that can exploit such a structure for the sake of good performance and low memory consumption.

Suppose we are fortunate and the input data is highly structured. For example, suppose the data has the schema of Figure 1 but is of much larger size, and suppose:

- enrollment is sorted by student_id and course_id and resides on disk, and

- course is sorted by course_id and resides in memory.

Then the example query can be executed with one scan of the enrollment table. This is accomplished by reading the enrollment table from disk. As each student appears, the course_id values associated with that student are merged with the course table. If all courses match, the student_id is copied to the result.

The single scan of the enrollment table is obviously the most efficient possible algorithm in this case. In the remainder of this paper, we will describe similar types of structure for input datasets, and the optimal algorithms that are associated with them. The notion of "optimality" will be further discussed in the next section.

Revisiting our example in Figure 1, how could this careful structuring of input data, such as sorting by student_id and course_id, occur? It could happen by chance, or for two other more commonly encountered reasons:

1. The data might be stored in tables, which were sorted in that order for other purposes, for example, so that it is easy to list enrollments on a roster in ID order, or to find course information when a course ID number is given.

2. The data might have undergone some previous processing, because the division operator query is part of a more complex query. The previous processing might have been a merge-join operator, for example, which requires that its inputs be sorted and produces sorted output data.

\subsection{Choice of Algorithms}

A query processor of a database system typically provides several algorithms that all realize the same operation. An optimizer has to choose one of these algorithms to process the given data. If the optimizer knows the structure of the input data for an operator, it can pick an algorithm that exploits the structure. Many criteria influence the decision why one algorithm is preferred over others. Some of these choice criteria are: the time to deliver the first/last result row, the amount of memory for internal, temporary data structures, the number of scans over the input data, or the ability to be non-blocking, i.e., to return some result rows before the entire input data are consumed.

Which algorithm should we use to process the division operation, given the dividend and divisor tables shown in Figure 1? Several algorithms are applicable but they are not equally efficient. For example, since the dividend and divisor are both sorted on the attribute course_id in Figure 1, we could select a division algorithm that exploits this fact by processing the input tuples in a way that is similar to the merge-join algorithm, as we have sketched in the previous section.

What algorithm should we select when the input tables are not sorted on course_id for each group of student_id? One option is to sort both input tables first and then employ the algorithm similar to merge-join. Of course, this incurs an additional computational cost for sorting in addition to the cost of the division algorithm itself. Another option is to employ an algorithm that is insensitive to the ordering of input tuples. One such well-known algorithm is hash-division and is discussed in detail in Section 3.3.4.

We have seen that the decision, which algorithm to select among a set of different division algorithms, depends on the structure of the input data. This situation is true for any class of algorithms, including those that implement database operators like join, aggregation, and sort algorithms.

It is possible that division is only a portion of a larger query that contains many additional query parts. Hence, the input of a division operation is not restricted to base tables, like in the example 
of Figure 1, but it can be derived tables which are the result of another operation like a join, for example. Furthermore, the output of the division could be an intermediate result itself that is further processed within the query. For example, the quotient table result in Figure 1 could be the input of an aggregation that counts the number of students. The meaning of the resulting aggregate is the number of students who have taken all courses of the department. Alternatively, the result in Figure 1 could be an input of a join with a table student(student_id, name, address,...) to retrieve a student's name, address, etc. instead of a meaningless ID. Thus, the result table produced by the selected division algorithm can have certain data properties that influence the choice of additional algorithms, here a join, that are used to process the overall query.

\subsection{Grouping}

Relational database systems have the notion of grouped rows in a table. Let us briefly look at an example that shows why grouping is important for query processing. Suppose we want to find for each course the number of enrolled students in the enrollment table of Figure 1. One way to compute the aggregates involves grouping: after the table has been grouped on course_id, all rows of the table with the same value of course_id appear next to each other. The ordering of the group values is not specified, i.e., any group of rows may follow any other group. Group-based aggregation groups the data first, and then it scans the resulting table once and computes the aggregates during the scan.

Another way to process this query is nestedloop aggregation. We pick any course ID as the first group value and then search through the whole table to find the rows that match this ID and compute the sum. Then, we pick a second course ID, search for matching rows, compute the second aggregate, pick the third value, etc. If no suitable search data structure (index) is available, this processing may involve multiple scans over the entire dataset.

The aggregation step of the group-based approach is obviously more efficient than the second approach because it can make an assumption about some ordering of the rows. However, the more efficient processing is paid with the overhead of the preceding grouping.

When a table is to be grouped on a list $\left(a_{1}, \ldots, a_{n}\right)$ of more than one attribute, the result is equal to grouping on a single attribute in an iterative way: We first group on $a_{1}$, then for each subset of rows defined by $a_{1}$, we group on $a_{2}$, and for each such subset determined by $a_{2}$, we group on $a_{3}$, etc. Hence, if we want to compare two tables that are grouped on the same set of attributes, we have to be aware of the attribute list ordering, because the resulting grouped table has a different structure for each ordering. This fact is important for division when we match some of the dividend's divisor attributes with all of the divisor's attributes.

Sorted data appears frequently in query processing. Note that sorting is a special grouping operation. For example, grouping only requires that students enrolled in the same course are stored next to each other (in any order), whereas sorting requires more effort, namely that they be in a particular order (ascending or descending). The overhead of sort-based grouping is reflected by the time complexity $O(n \log n)$ as opposed to the nearly linear time complexity for hashbased grouping. Though sort-based grouping algorithms do more than necessary, both hashbased and sort-based grouping perform well for large datasets $[7,8]$.

\subsection{Grouped Input Data for Division}

Relational division has two input tables, a dividend and a divisor, and it returns a quotient table. As a consequence of the definition of the division operator, we can partition the attributes of the dividend $S$ into two sets, which we denote $D$ and $Q$, because they correspond to the attributes of the divisor and the quotient, respectively. The divisor's attributes correspond to $D$, i.e., for each attribute in the divisor there is a different attribute in $D$ of the same domain. As already mentioned, for simplicity, we assume that the names of attributes in the quotient $R$ are the same as the corresponding attribute names in the dividend $S$ and the divisor $T$. Thus, we write a division operation as $R(Q)=S(Q \cup D) \div T(D)$. In 
Figure $1, Q=\{$ student_id $\}$ and $D=\{$ course_id $\}$.

Our classification of division algorithms is based on whether certain attributes are grouped or even sorted. Several reasons justify this decision. Grouped input can reduce the amount of memory needed by an algorithm to temporarily store rows of a table because all rows of a group have a constant group value. Furthermore, grouping appears frequently in query processing. Many database operators require grouped or sorted input data (e.g., merge-join) or produce such output data (e.g., index-scan): If there is an index defined on a base table, a query processor can retrieve the rows in sorted order, specified by the index attribute list. Thus, in some situations algorithms may exploit for the sake of efficiency the fact that base tables or derived tables are grouped if the system knows about this fact.

In Table 1, we show all possible classes of input data based on whether or not interesting attribute sets are grouped, i.e., grouped on one of $Q, D$, or the divisor. As we will see later in this paper, some classes have no suitable algorithm that can exploit its specific combination of data properties. The classes that have at least one algorithm exploiting exactly its data properties are shown in bold font. In class 0 , for example, no table is grouped on an interesting attribute set. Algorithms for this class have to be insensitive to whether the data is grouped or not. Another example scenario is class 10. Here, the dividend is first grouped on the quotient attributes $Q$ (denoted by $G_{1}$, the major group) and for each group, it is grouped on the divisor $D$ (denoted by $G_{2}$, the minor group). The divisor is grouped in the same ordering $\left(G_{2}\right)$ as the dividend.

Our classification is based on grouping only. As we have seen, some algorithms may require that the input is even sorted and not merely grouped. We consider this a minor special case of our classification, so we do not reflect this data property in Table 1, but the algorithms in Section 3 will refer to this distinction. We do not consider any data property other than grouping in this paper because our approach is complete and can easily and effectively be exploited by a query optimizer and query processor.

enrollment
\begin{tabular}{|l|l|}
\hline student_id & course_id \\
\hline \hline Bob & Theory \\
\hline Alice & Compilers \\
\hline Chris & Theory \\
\hline Chris & Graphics \\
\hline Alice & Theory \\
\hline Bob & Graphics \\
\hline Chris & Compilers \\
\hline Bob & Databases \\
\hline Bob & Compilers \\
\hline not grouped & not grouped
\end{tabular}

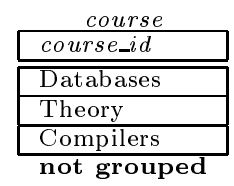

(a) Class 0

\begin{tabular}{|l|l|}
\hline \multicolumn{2}{|c|}{ enrollment } \\
\hline \hline Alice & course_id \\
\hline Chris & Theory \\
\hline Bob & Theory \\
\hline Bob & Theory \\
\hline Bob & Databases \\
\hline Chris & Graphics \\
\hline Bob & Graphics \\
\hline Chris & Compilers \\
\hline Alice & Compilers \\
\hline not grouped & Compilers \\
\hline
\end{tabular}

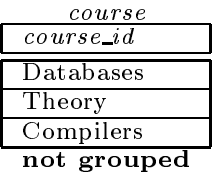

(b) Class 2

enrollment
\begin{tabular}{|l|l|}
\hline student_id & course_id \\
\hline \hline Chris & Graphics \\
\hline Chris & Compilers \\
\hline Chris & Theory \\
\hline Alice & Theory \\
\hline Alice & Compilers \\
\hline Bob & Theory \\
\hline Bob & Compilers \\
\hline Bob & Databases \\
\hline Bob & Graphics \\
\hline grouped & not grouped
\end{tabular}

(c) Class 5

\begin{tabular}{|c|c|}
\hline student_id & course_id \\
\hline Chris & Theory \\
\hline Chris & Graphics \\
\hline Chris & Compilers \\
\hline Alice & Theory \\
\hline Alice & Compilers \\
\hline Bob & Databases \\
\hline$\overline{B o b}$ & Theory \\
\hline Bob & Graphics \\
\hline Bob & Compilers \\
\hline
\end{tabular}

course

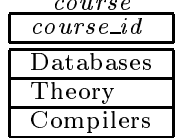

grouped

Figure 2. Four important classes of input data, based on the example of Figure 1

Figure 2 illustrates four classes of input data for division, based on the example data of Figure 1. These classes, which are shown in bold font 


\begin{tabular}{|c|c|c|c|l|}
\hline Class & \multicolumn{2}{|c|}{ Dividend } & Divisor & Description of Grouping \\
\cline { 2 - 5 } & $Q$ & $D$ & & \\
\hline \hline $\mathbf{0}$ & $N$ & $N$ & $N$ & \\
\hline 1 & $N$ & $N$ & $G$ & \\
\hline $\mathbf{2}$ & $N$ & $G$ & $N$ & \\
\hline 3 & $N$ & $G_{1}$ & $G_{2}$ & arbitrary ordering of groups in $D$ and divisor \\
\hline 4 & $N$ & $G_{1}$ & $G_{1}$ & same ordering of groups in $D$ and divisor \\
\hline $\mathbf{5}$ & $G$ & $N$ & $N$ & \\
\hline 6 & $G$ & $N$ & $G$ & \\
\hline 7 & $G_{1}$ & $G_{2}$ & $N$ & $Q$ major, $D$ minor \\
\hline 8 & $G_{2}$ & $G_{1}$ & $N$ & $D$ major, $Q$ minor \\
\hline 9 & $G_{1}$ & $G_{2}$ & $G_{3}$ & $Q$ major, $D$ minor; arbitrary ordering of groups in $D$ and divisor \\
\hline $\mathbf{1 0}$ & $G_{1}$ & $G_{2}$ & $G_{2}$ & $Q$ major, $D$ minor; same ordering of groups in $D$ and divisor \\
\hline 11 & $G_{2}$ & $G_{1}$ & $G_{3}$ & $D$ major, $Q$ minor; arbitrary ordering of groups in $D$ and divisor \\
\hline 12 & $G_{2}$ & $G_{1}$ & $G_{1}$ & $D$ major, $Q$ minor; same ordering of groups in $D$ and divisor \\
\hline
\end{tabular}

Table 1

A classification of dividend and divisor. Attributes are either grouped $(G)$ or not grouped $(N)$. We use the same (a different) subscript of $G$ when $D$ and the divisor have the same (a different) ordering of groups in classes $3,4,9-12$. In addition, when the dividend is grouped on both $Q$ and $D$ in classes 7-12, then $G_{1}\left(G_{2}\right)$ denotes the attributes that the table is grouped on first (second).

in Table 1, are important for several algorithms that we present in the following section. Notice that for class 10 both tables are grouped in the same order on course_id. If the value "Graphics" is present in a quotient group then it always appears after "Theory" and before "Compilers." Figure 1 shows another example instance of class 10 , where the quotient order as well as the divisor group order is ascending. The benefit of knowing about such an input data property will be clarified when we discuss algorithms exploiting this specific property in Sections 3.3.2 and 3.3.3.

If we know that an algorithm can process data of a specific class, it is useful to know which other classes are also covered by the algorithm. This information can be represented, e.g., by a Boolean matrix like the one on the left in Figure 3. One axis indicates a given class $C_{1}$ and the other axis shows the other classes $C_{2}$ that are also covered by $C_{1}$. Alternatively, we can use a directed acyclic graph representing the input data classification, sketched on the right of Figure 3. If a cell of the matrix is marked with "Y" (yes), or equivalently, if there is a path in the graph

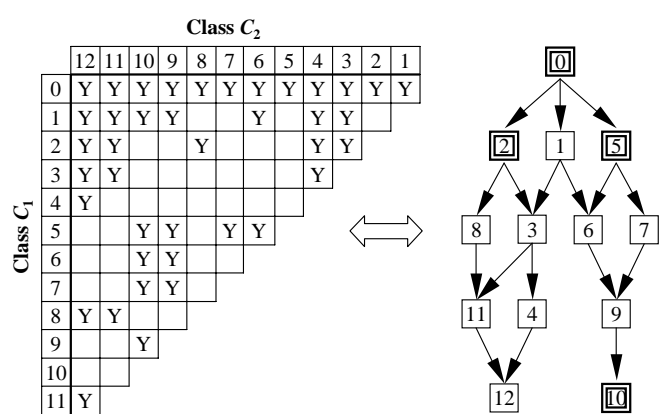

Figure 3. A matrix and a directed acyclic graph representing the input data classification described in Table 1. All algorithms to be discussed in Section 3 assume data properties of either class $0,2,5$, or 10 .

from class $C_{1}$ to $C_{2}$, then an algorithm that can process data of class $C_{1}$ can also process data of class $C_{2}$. The graph clearly shows that the classification is a partial order of classes, not a strict hierarchy. The source node of the graph is class 


\begin{tabular}{|l|l|}
\hline \multicolumn{1}{|c|}{ Division Algorithm } & Abbrev. \\
\hline \hline Hash-Division & HD \\
\hline Hash-Division for Divisor Groups & HDD \\
\hline Hash-Division for Quotient Groups & HDQ \\
\hline Merge-Count Division & MCD \\
\hline Merge-Group Division & MGD \\
\hline Merge-Sort Division & MSD \\
\hline Nested-Loops Division & NLD \\
\hline Nested-Loops Counting Division & NLCD \\
\hline Transposed Hash-Division & HDT \\
\hline Transposed Hash-Division for Divisor Groups & HDTD \\
\hline Transposed Hash-Division for Quotient Groups & HDTQ \\
\hline Stream-Join Division & SJD \\
\hline
\end{tabular}

Table 2

Abbreviations for division algorithms

0 , which requires no grouping of $D, Q$, or divisor. Any algorithm that can process data of class 0 can process data of any other class. For example, an algorithm processing data of class 6 is able to process data of classes 9 and 10 .

For the subsequent discussion of division algorithms, we define two terms to refer to certain row subsets of the dividend. Let the dividend $S$ be grouped on $Q(D)$ as the first or the only set of group attributes, i.e., let the dividend belong to class 5 (2) and all its descendants in Figure 3. Furthermore, let $v$ be one specific value of such a group. Then, the set of rows defined by $\sigma_{Q=v}(S)$ $\left(\sigma_{D=v}(S)\right)$ is called the quotient group (divisor group) of $v$. For example, in the enrollment table of class 5 in Figure 2(c), the quotient group of Alice consists of the rows $\{$ (Alice, Theory), (Alice, Compilers) $\}$. Similarly, the divisor group of Databases in class 2 in Figure 2(b) consists of the single row (Bob, Databases).

\section{Overview of Algorithms}

In this section, we present algorithms for relational division proposed in the database literature together with several new variations of the wellknown hash-division algorithm. For the sake of a concise presentation, we will frequently use abbreviations for the algorithms that we summarize in Table 2.

In Section 4, we will analyze and compare the effectiveness of each algorithm with respect to the data classification of Section 2.

\subsection{Complexity of Algorithms}

During the evaluation of relevant literature, we found that it is necessary to clarify that each division algorithm (analogous to other classes of algorithms, like joins, for example) has performance advantages for certain data characteristics. No algorithm is able to outperform the others for every input data conceivable.

The following algorithms assume that the division's input consists of a dividend table $S(Q, D)$ and a divisor table $T(D)$, where $Q$ is a set of quotient attributes and $D$ is the set of divisor attributes, as defined in Section 2.4.

During the presentation of the algorithms, we analyze the worst and typical case complexities of processing time and memory consumption in $O$-notation, based on the size (number of rows) of the dividend $|S|$ and the size of the divisor $|T|$. We use $|Q|$, the number of distinct values of quotient attributes $Q$ in the dividend, for some algorithms to derive a complexity formula. Note that always $|Q| \leq|S|$, and in the worst case $|Q|=|S|$, i.e., each single row of $S$ is a potential (candidate) quotient. To derive formulas for the typical time and memory complexities, we use the assumption that $|S| \gg|T|$, i.e., there are many quotient candidates and/or the number of rows of an typical quotient candidate is much larger than the number of divisor rows. We consider this situation as the typical case because relational division is defined to compute a set of result rows and in realworld scenarios this set is of considerable size. A large result size occurs only if the dividend contains many more rows than the divisor.

In addition to time and memory complexity, it is useful to analyze the I/O cost of each algorithm, as it has been done in detail for some of the following algorithms in [8]. However, since the focus of this paper is to describe the fundamental structure of input data and algorithms involved in relational division, we restrict our analysis to memory and processing complexities and we do not give $\mathrm{I} / \mathrm{O}$ formulas.

\subsection{Query Language Representation and Algorithm Classification}

In this section, we show SQL expressions for division and explain how they give rise to two 
classes of algorithms based on the kind of data structures employed.

The commonly used approach to express universal quantification uses two "NOT EXISTS" clauses, exploiting the mathematical equivalence $\forall x \exists y: f(x, y) \equiv \neg \exists x \neg \exists y: f(x, y)$ as follows:

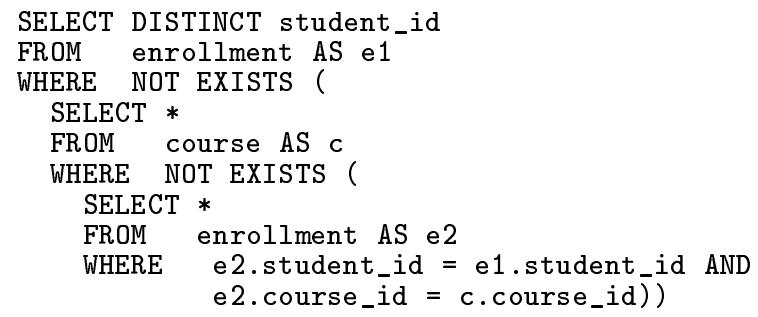

This query asks for each student, where there is no course that the student is not enrolled in.

The previous approach is not very intuitive to formulate. Another way to express division queries has been proposed in the past, using a special syntax for universal quantification. The quantifier "FOR ALL," which is part of a socalled quantified predicate [9], was planned to be included in the SQL:1999 standard [2] but it was finally excluded for reasons unknown to the authors. We can phrase queries using the quantifier for division queries in an intuitive way. For example, the following SQL query employing a quantified predicate is equivalent to the above query:

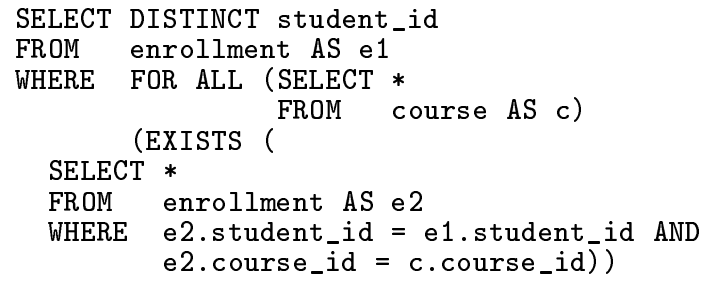

This query asks for each student, where for all courses there is an enrollment of this student.

A query language syntax dedicated to universal quantification allows us to map the query directly to a query execution that uses a division algorithm. It is nontrivial to map a query formulated in an indirect way (e.g. by using nested negations as in the first approach) to a query execution that uses a division algorithm.

There is a third way mentioned in the literature that uses aggregation. The example query of Section 1.1 can be phrased in SQL using aggregation as follows:

\begin{tabular}{|c|c|c|}
\hline \multicolumn{2}{|c|}{ old_enrollment } & \multirow[b]{4}{*}{ course } \\
\hline student_id & course_id & \\
\hline Chris & Compilers & \\
\hline Chris & Graphics & \\
\hline Chris & Theory & course_id \\
\hline Alice & Compilers & Databases \\
\hline Alice & Theory & Compilers \\
\hline Bob & Compilers & Theory \\
\hline Bob & Databases & (b) Divisor \\
\hline Bob & Graphics & \\
\hline Bob & Theory & \\
\hline
\end{tabular}

(a) Original dividend

new_enrollmen

\begin{tabular}{|l|l|}
\hline student_id & course_id \\
\hline \hline Chris & Compilers \\
\hline Chris & Theory \\
\hline Alice & Compilers \\
\hline Alice & Theory \\
\hline Bob & Compilers \\
\hline Bob & Databases \\
\hline Bob & Theory \\
\hline
\end{tabular}

(c) Resulting dividend

Figure 4. Semi-join old_enrollment $\ltimes$ course $=$ new_enrollment, representing the preprocessing of the enrollment table for aggregate division algorithms, based on the example in Figure 1.

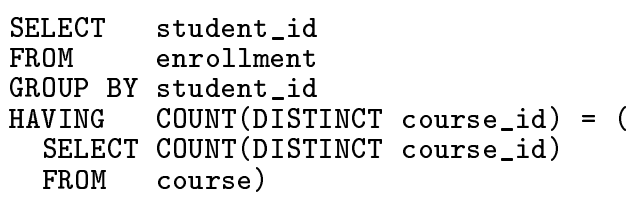

Any query involving universal quantification can be replaced by a query that makes use of counting [8]. However, there is a problem with this approach to express division because it is not equivalent to the previous two approaches. It returns the same result as the other queries only if two conditions are met. First, each course_id $(D)$ value in enrollment (the dividend) is also contained in the course table (the divisor). Defining a foreign key enrollment.course_id that references course and enforcing referential integrity can fulfill this condition. Another way to guarantee referential integrity is to preprocess the dividend by a semi-join of dividend and divisor. The semijoin returns all dividend rows whose $D$ values are contained in the divisor. Figure 4 illustrates the semi-join for our university example in Figure 1. 
The second condition of this approach requires that the course_id $(D)$ values and the divisor rows are unique. Possible duplicates have to be removed before the division. Hence, the SQL query above contains the SQL keyword "DISTINCT" when counting course_id values to avoid any duplicates. Note that when the divisor is grouped on all of its attributes, each group consists of a single row because of the required absence of duplicate rows. The same is true for the dividend if it is grouped on both $Q$ and $D$, as in the classes 7-12 in Table 1.

We have seen that the two approaches actually realize two logical operators that give rise to two classes of algorithms, aggregate and scalar. The scalar class of algorithms relies on direct row matches between the dividend's divisor attributes $D$ and the divisor table. The second class, aggregate algorithms, use counters to compare the number of rows in a dividend's quotient group to the number of divisor rows. In [3], scalar and aggregate algorithms are called direct and indirect algorithms, respectively.

Aggregate algorithms are often described as alternative ways to scalar algorithms (for the real division operator) but they are prone to errors because one has to take care of duplicates, NULL values, and referential integrity, as already mentioned before.

Some query languages for non-relational data models also offer support to express quantification. For example, there is "work in progress" by the W3C on the Working Draft of XQuery [28], a query language for XML data. Universal quantification can be expressed in XQuery by an every expression.

\subsection{Scalar Algorithms}

This section presents division algorithms that use data structures to directly match dividend rows with divisor rows.

\subsubsection{Nested-Loops Division}

This algorithm is the most naïve way to implement division. However, like nested-loops join, an operator using nested-loops division (NLD) has no required data properties on the input tables and thus can always be employed, i.e., NLD can pro- cess input data of class 0 and thus any other class of data, according to Figure 3.

We use two set data structures, one to store the set of divisor values of the divisor table, called seen_divisors, and another to store the set of quotient candidate values that we have found so far in the dividend table, called seen_quotients. We first scan the divisor table to fill seen_divisors. After that, we scan the dividend in an outer loop. For each dividend row, we check if its quotient value $(Q)$ is already contained in seen_quotients. If not, we append it to the seen_quotients data structure and scan the remainder of the dividend iteratively in an inner loop to find all rows that have the same quotient value as the dividend row of the outer loop. For each such row found, we check if its divisor value is in seen_divisors. If yes, we mark the divisor value in seen_divisors. After the inner scan is complete, we add the current quotient value to the output if all divisors in seen_divisors are marked. Before we start processing the next dividend row of the outer loop, we unmark all elements of seen_divisors.

Note that NLD can be very inefficient. For each row in the dividend table, we scan the dividend at least partially to find all the rows that belong to the current quotient candidate. All divisor rows and quotient candidate rows are stored in an inmemory data structure. NLD can be the most efficient algorithm for small ungrouped datasets.

This algorithm can make use of any set data structure like hash tables or sorted lists to represent seen_divisors and seen_quotients. Let us assume that this algorithm uses hash tables or any very efficient data structure with a (nearly) constant access time. Then, the worst case time complexity of this algorithm is $O\left(|S|^{2}+|T|\right)$ and the typical time complexity is $O\left(|S|^{2}\right)$. The memory complexity is $O(|Q|+|T|)$. Since in the extreme case $|Q|=|S|$, the worst case memory complexity is $O(|S|+|T|)$ and the typical memory complexity is $O(|S|)$.

The pseudo code of the nested-loops division algorithm is shown in the Appendix. In that code, the seen_divisors and seen_quotients data structures are represented by the divisor hash table $d h t$ and the quotient hash table $q h t$, respectively.

Figure $5(\mathrm{~g})$ illustrates the two hash tables 


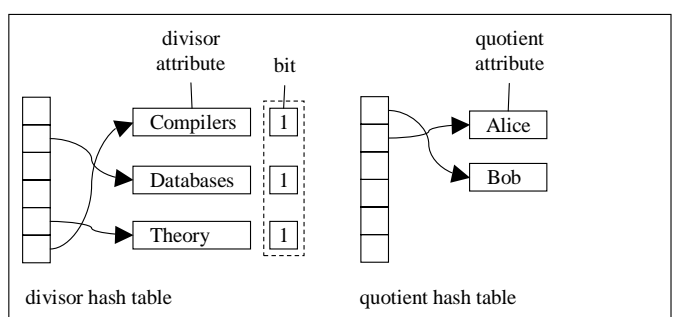

(a) Nested-Loops Division (NLD)

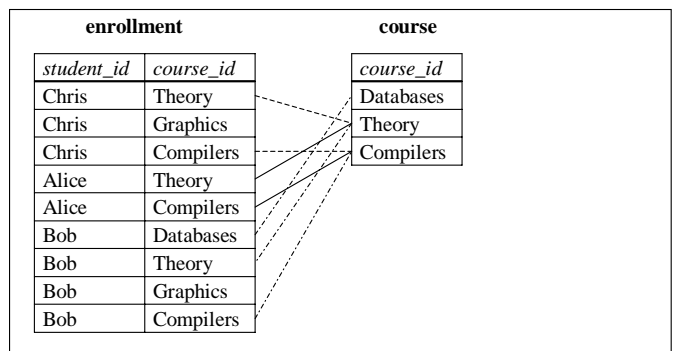

(c) Merge-Group Division (MGD)

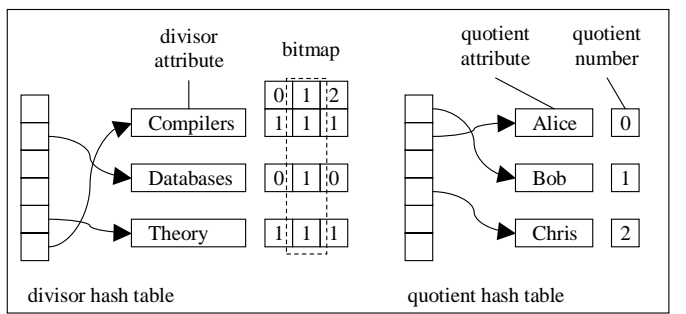

(e) Transposed Hash-Division (HDT)

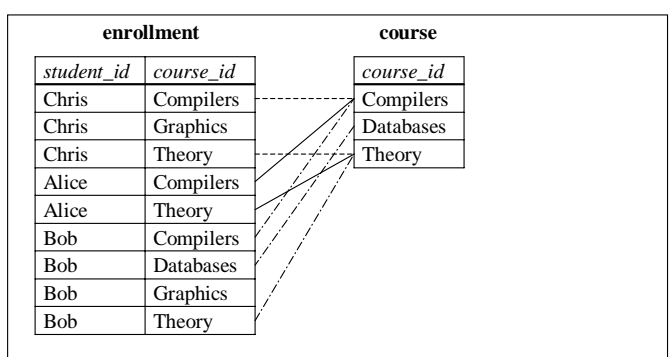

(b) Merge-Sort Division (MSD)

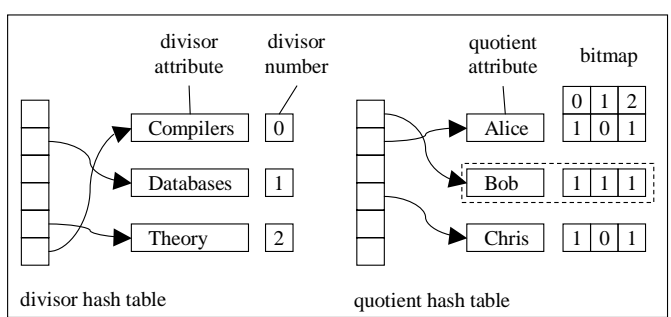

(d) Hash-Division (HD)

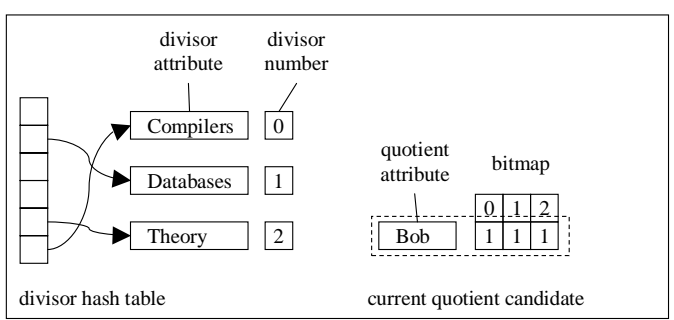

(f) Hash-Division for Quotient Groups (HDQ)

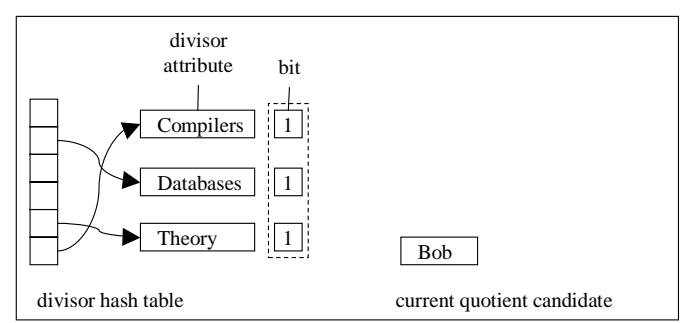

(g) Transposed Hash-Division for Divisor Groups (HDTQ)

Figure 5. Overview of the data structures and processing used in scalar algorithms. The value setting is based on the example from Figure 1. Except for MSD and MGD, broken lined boxes indicate that a quotient is found.

used in this algorithm: the divisor/quotient hash table represents seen_divisors/seen_quotients, respectively. The value setting in the hash tables is shown for the time when all dividend rows of Alice and Bob (in this order) have been processed and we have not yet started to process any rows 
of Chris in the outer loop. We find that Bob is a quotient because all bits in the divisor hash table are equal to 1 .

\subsubsection{Merge-Sort Division}

The merge-sort division (MSD) algorithm assumes that

- the divisor $T$ is sorted, and that

- the dividend $S$ is grouped on $Q$, and for each group, it is sorted on $D$ in the same order (ascending or descending) as $T$.

This data characteristic is a special case of class 10 , where $D$ and the divisor are sorted and not only grouped.

The algorithm resembles merge-join for processing a single quotient group and is similar to nested-loops join for processing all groups. Let us briefly sketch the processing of rows within a single group, assuming an ascending sort order. We begin with the first row of dividend and divisor. If the divisor value $D$ of the current dividend row and the divisor row match, we proceed with the next row in both tables. If $D$ is greater than the current divisor row, we scan forward to the next quotient group. If $D$ is less than the divisor row, we proceed with the next row of the group and the current divisor row. If there are no more rows to process in the quotient group but at least one more row in the divisor, we skip the quotient group. If there are no more rows to process in the divisor, we have found a quotient and add it to the output table.

Our merge-sort division is similar to the approach called naïve division, presented in [8] and originating from [26]. In both approaches, we can implement the scan of each input such that it ignores duplicates. In contrast to merge-sort division, naïve division explicitly sorts the data before the merge step. Even worse, naïve division does not merely group the dividend on $Q$ but sorts it, which is more than necessary. Note that we view sorting or grouping as preprocessing activities that are separate from the core division algorithm. We sketch the pseudo code of mergesort division without duplicate removal logic in the Appendix.
The worst case time complexity of this algorithm is $O(|S|+|Q||T|)=O(|S|+|S||T|)=$ $O(|S||T|)$ because the dividend is scanned exactly once and from the divisor table, we fetch as many rows as the number of quotient candidates times the number of divisor rows. The typical case time complexity is $O(|S||T|)$. The worst and typical case memory complexity is $O(1)$, since only a constant number of small data structures (two rows) have to be kept in memory.

Figure 5(b) illustrates the matches between rows of dividend and divisor. Observe that the data is not sorted but only grouped on student_id in an arbitrary order.

\subsubsection{Merge-Group Division}

We can generalize merge-sort division to an algorithm that we call merge-group division (MGD). In contrast to MSD, we assume that

- both inputs are only grouped and not necessarily sorted on the divisor attributes, but that

- the order of groups in each quotient group and the order of groups in the divisor are the same.

Note that each group within a quotient group and within the divisor consists of a single row. This ordering can occur (or can be achieved) if, e.g., the same hash function is used for grouping the divisor and each quotient group.

In the MSD algorithm, we can safely skip a quotient candidate if the current value of $Q$ is greater (less) than that of the current divisor row, assuming an ascending (a descending) sort order. Since we do not require a sort order on these attributes in MGD, we cannot skip a group on unequal values, as we do in MSD. For example, suppose that the divisor $S$ has a single integer attribute and consists of the following rows in the given order: $S=(3,1,5)$ and the $D$ values of the current quotient group $G$ consists of the rows $G=(2,5,4,6)$. We can be sure that $G$ is not a valid quotient only after

- we have scanned the entire group $G$, where we find that the first element of $S(3)$ is not contained in $G$, or 
- we have scanned $S$ up to last element (5) and we have scanned $G$ up to the second element (5) to find that $G$ does not contain the other elements of $S$ (3 and 1) before element 5 appears.

The MGD approach makes use of a look-ahead of $n$ divisor rows for some predefined value $n \geq 1$. As in the MSD approach, we compare the current quotient group row with the current divisor row. In case of inequality, we look ahead up to the $n$-th divisor row to see if there is any other row matching the current group row. If we find such a match, we can skip the current quotient candidate. In our example, a look-ahead of 2 means that we check up to the second element (1) of the divisor. The look-ahead of 2 does not help for any value of $G$ in our example. A look-ahead of 3 means a check with up to the third divisor element (5). When we check the second row (5) of the quotient group, we find a match with the third divisor element (5). Here, we can skip the group because a quotient would have to contain the values 3 and 1 before the occurrence of 5 to qualify due to the assumption that the group orders are the same. In other words, the ordering assumption guarantees that the values 3 and 1 cannot occur after the element 5 . Since they have neither occurred in $G$ before element 5, we know that this quotient candidate does not contain all divisor elements, in particular not the elements 3 and 1 .

The MSD algorithm is a special case of MGD where the look-ahead is set to 1 because it does not look further than the current row for each quotient group row since sorting was applied.

In summary, the MGD approach can make use of as much look-ahead as the minimum of the available memory and the current divisor size. Note that the divisor fits into memory in all reasonable cases. Figure 5(c) sketches the matches between dividend and divisor rows. Observe that the order of (single-row) groups within each quotient group in the dividend is the same as that of the divisor.

The time complexity of this algorithm is $O(|S|+|Q||T|)$ because the dividend is scanned exactly once and the divisor is scanned entirely for each quotient and at least partially for every quotient candidate. Thus, the worst case time complexity is $O(|S|+|S||T|)=O(|S||T|)$. The typical case time complexity is also $O(|S||T|)$. The worst case memory complexity is $O(|T|)$ if we keep the entire divisor as a look-ahead in memory. The typical case memory complexity then becomes $O(1)$ since $|T| \ll|S|$.

\subsubsection{Classic Hash-Division}

In this section, we present the classic hashdivision (HD) algorithm [8]. We call this algorithm "classic" to distinguish it from our variations of this approach in the following sections.

The two central data structures of HD are the divisor and quotient hash tables, sketched in Figure $5(\mathrm{~d})$. The divisor hash table stores divisor rows. Each such row has an integer value, called divisor number, stored together with it. The quotient hash table stores quotient candidates and has a bitmap stored together with each candidate, with one bit for each divisor. The pseudo code of hash-division is sketched in the Appendix.

In a first phase, hash-division builds the divisor hash table while scanning the divisor. The hash function takes the divisor attributes as an argument and assigns a hash bucket to each divisor row. A divisor row is stored into the hash bucket only if it is not already contained in the bucket, thus eliminating duplicates in the divisor. When a divisor row is stored, we assign a unique divisor number to it by copying the value of a global counter. This counter is incremented for each stored divisor row and is initialized with zero. The divisor number is used as an index for the bitmaps of the quotient hash table.

The second phase of the algorithm constructs the quotient hash table while scanning the dividend. For each dividend row, we first check if its $D$ value is contained in the divisor hash table, using the same hash function as before. If yes, we look up the associated divisor number, otherwise we skip the dividend row. In addition to the look-up, we check if the quotient is already present in the quotient hash table. If yes, we update the bitmap associated with the matching quotient row by setting the bit to 1 whose position is equal to the divisor number we looked 
up. Otherwise, we insert a new quotient row into the quotient hash table together with a bitmap where all bits are initialized with zeroes and the appropriate bit is set to 1 , as described before. Since we insert only quotient candidates that are not already contained in the hash table, we avoid duplicate dividend rows.

The final phase of hash division scans the quotient hash table's buckets and adds all quotient candidates to the output whose bitmaps contain only ones. In Figure 5(d), the contents of the hash tables are shown for the time when all dividend and divisor rows of Figure 1 have been processed. We see that since Bob's bitmap contains no zeroes, Bob is the only quotient, indicated by a broken lined box.

Hash-division scans both dividend and divisor exactly once. Because hash tables are employed that have a nearly constant access time, this approach has a worst and typical case time complexity of $O(|S|+|T|)$ and $O(|S|)$, respectively. The memory complexity consists of $O(|T|)$ to store the divisor hash table plus $O(|Q||S|)$ for the quotient hash table. The size of a bitmap is proportional to $|S|$. Since the worst case scenario implies that $|Q|=|S|$, the total worst and typical case memory complexity is $O(|S||T|)$.

\subsubsection{Transposed Hash-Division}

This algorithm is a slight variation of classic hash-division. The idea is to switch the roles of the divisor and quotient hash tables. The transposed hash-division (HDT) algorithm keeps a bitmap together with each row in the divisor hash table instead of the quotient hash table, as in HD. Furthermore, HDT keeps an integer value with each row in the quotient hash table instead of the divisor hash table, as in the HD algorithm.

Same as the classic hash-division algorithm, HDT first builds the divisor hash table. However, we store a bitmap with each row of the divisor. A value of 1 at a certain bit position of a bitmap indicates which quotient candidate has the same values of $D$ as the given divisor row.

In a second phase, also same as HD, the HDT algorithm scans the dividend table and builds a quotient hash table. For each dividend row, the $D$ values are inserted into the divisor hash table as follows. If there is a matching quotient row stored in the quotient hash table, we look up its quotient number. Otherwise, we insert a new quotient row together with a new quotient number. Then, we update the divisor row's bitmap by setting the bit at the position given by the quotient number to 1.

The final phase makes use of a new, separate bitmap, whose size is the same as the bitmaps in the divisor hash table. All bits of the bitmap are initialized with zero. While scanning the divisor hash table, we apply a bit-wise AND operation between each bitmap contained and the new bitmap. The resulting bit pattern of the new bitmap is used to identify the quotients. The quotient numbers (bit positions) with a value of 1 are then used to look up the quotients using a quotient vector data structure that allows a fast mapping of a quotient number to a quotient candidate. The HDT pseudo code is shown in the Appendix.

Figures 5(d) and (e) contrast the different structure of hash tables in HD and HDT. The hash table contents is shown for the time when all enrollment rows of Figure 1 have been processed. While a quotient in the HD algorithm can be added to the output when the associated bitmap contains no zeroes, the HDT algorithm requires a match of the bit at the same position of all bitmaps in the divisor table and it requires in addition a look-up in the quotient hash table to find the associated quotient row.

The time and memory complexities of HDT are the same as those of classic hash-division.

\subsubsection{Hash-Division for Quotient Groups}

Both, classic and transposed hash-division can be improved if the dividend is grouped on either $D$ or $Q$. However, our optimizations based on divisor groups lead to aggregate, not scalar algorithms. Hence, this section on scalar algorithms presents some optimizations for quotient groups. The optimizations of hash-division for divisor groups are presented in Section 3.4.3.

Let us first focus on classic hash-division. If the dividend is grouped on $Q$, we do not need a quotient hash table. It suffices to keep a single bitmap to check if the current quotient candidate 
is actually a quotient. When all dividend rows of a quotient group have been processed and all bits of the bitmap are equal to 1 , the quotient row is added to the output. Otherwise, we reset all bits to zero, skip the current quotient row, and continue processing the next quotient candidate. Because of the group-by-group processing of the improved algorithm, we call this approach hashdivision for quotient groups (HDQ).

The HDQ algorithm is non-blocking because we return a quotient row to the output as soon as a group of (typically few) dividend rows has been processed. In contrast, the HD algorithm has a final output phase: the quotient rows are added to the result table after the entire dividend has been processed because hash-division does not assume a grouping on $Q$. For example, the "first" and the "last" row of the dividend could belong to the same quotient candidate, hence the HD algorithm has to keep the state of the candidate quotient row as long as at least one bit of the candidate's bitmap is equal to zero. Note that it is possible to enhance HD such that it is not a "fully" blocking algorithm. If bitmaps are checked during the processing of the input, HD could detect some quotients that can be returned to the output before the entire dividend has been scanned. Of course, we would then have to make sure that no duplicate quotients are created, either by preprocessing or by referential integrity enforcements or by keeping the quotient value in the hash table until the end of the processing. In this paper, we do not elaborate on this variation of HD.

\subsubsection{Transposed Hash-Division for Quo- tient Groups}

We have seen that the HDQ algorithm is a variation of the HD algorithm: if the dividend is grouped on $Q$, we can do without a quotient hash table. Exactly the same idea can be applied to HDT yielding an algorithm that we call transposed hash-division for quotient groups (HDTQ).

For grouped quotient attributes, we can do without the quotient hash table and we do not keep long bitmaps in the divisor hash table but only a single bit per divisor. Before any group is processed, the bit of each divisor attribute is set to zero. For each group, we process the rows like in the HDT algorithm. After a group is processed, we add a quotient to the output if the bit of every divisor row is equal to 1 . Then, we reset all bits to zero and resume the dividend scan with the next group.

We do not show the pseudo code for the HDQ and HDTQ algorithms for brevity. However, we sketch their data structures in the Figures 5 (f) and $(\mathrm{g})$ for the time when the group of dividend rows containing the quotient candidate Bob have been processed.

\subsection{Aggregate Algorithms}

This class of algorithms compares the number of rows in each quotient candidate with the number of divisor rows. In case of equality, a quotient candidate becomes a quotient. All algorithms have in common that in a first phase, the divisor table is scanned once to count the number of divisor rows. Each algorithm then uses different data structures to keep track of the number of rows in a quotient candidate. Some algorithms assume that the dividend is grouped on $Q$ or $D$.

\subsubsection{Nested-Loops Counting Division}

Similar to scalar nested-loops division, nestedloops counting division (NLCD) is the most naïve way in the class of aggregate algorithms. This algorithm scans the dividend multiple times. During each scan, NLCD counts the number of rows belonging to the same quotient candidate.

We have to keep track of which quotient candidates we have already checked, using a quotient hash table as shown in Figure 6(a). A global counter is used to keep track of the number of dividend rows belonging to the same quotient candidate. We fully scan the dividend in an outer loop: We pick the first dividend row, insert its $Q$ value into the quotient hash table, and set the counter to 1 . If the counter's value is equal to the divisor count, we add the quotient to the output and continue with the next row of the outer loop. Otherwise, we scan the dividend in an inner loop for rows with the same $Q$ value as the current quotient candidate. For each such row, the counter is checked and in case of equality, the quotient is added to the output. When the end of the dividend is reached in the inner loop, we 


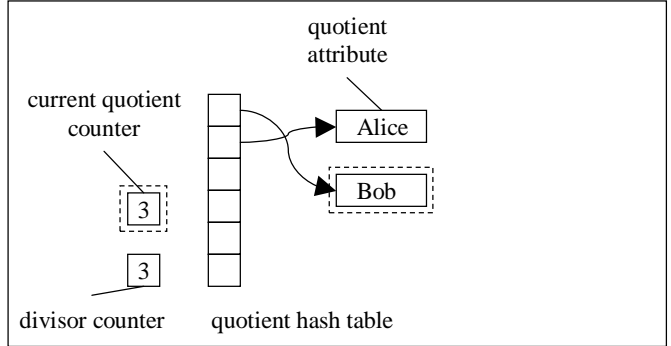

(a) Nested-Loops Counting Division (NLCD)

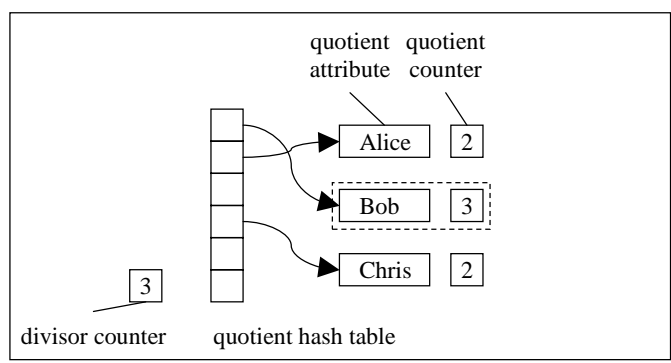

(c) Hash-Division for Divisor Groups (HDD)

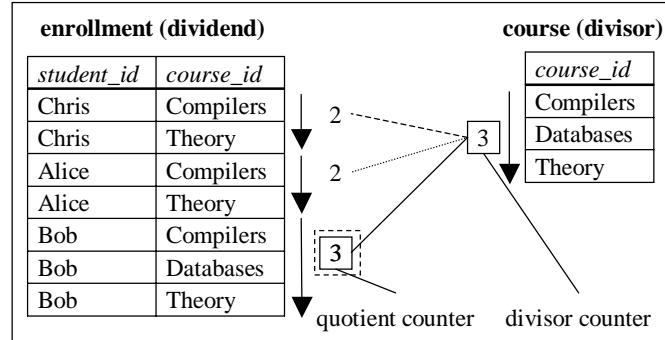

(b) Merge-Count Division (MCD)

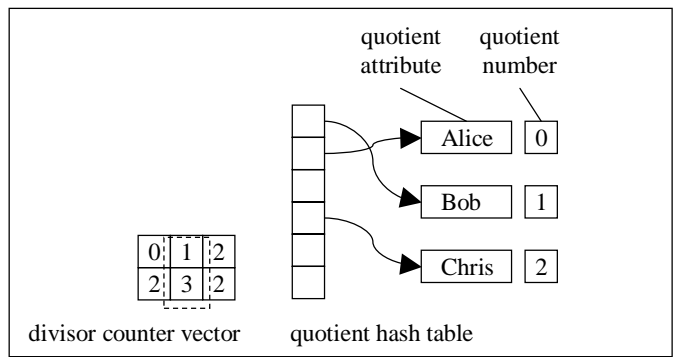

(d) Transposed Hash-Division for Divisor Groups (HDTD)

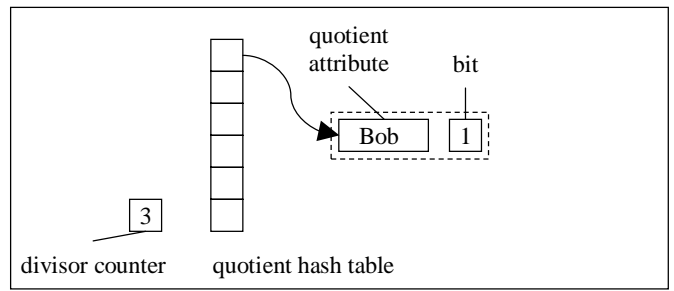

(e) Stream-Join Division (SJD)

Figure 6. Overview of data structures used in aggregate algorithms. Broken lined boxes indicate that a quotient is found. Only Bob's group has as many dividend rows as the divisor.

continue with the next row of the outer loop and check the hash table if this new row is a new quotient candidate.

The time and memory complexities are the same as for nested-loops division.

\subsubsection{Merge-Count Division}

Assuming that the dividend is grouped on $Q$, merge-count division (MCD) scans the dividend exactly once. After a quotient candidate has been processed and the number of rows is equal to those of the divisor, the quotient is added to the output. Note that the size of a quotient group cannot exceed the number of divisor groups because we have to guarantee referential integrity.

The aggregate algorithm merge-count division is similar to the scalar algorithms MSD and MGD, described in Sections 3.3.2 and 3.3.3. Instead of comparing the elements of quotient groups with the divisor, MCD uses a representative (the row count) of each quotient group to compare it with the divisor's aggregate. Figure 6(b) illustrates the single scan required to compare the size of the each quotient group with the divisor size.

MCD has a worst case time complexity of 
$O(|S|+|T|)$ and an typical case time complexity of $O(|S|)$. Since no significant data structures have to be kept in memory except for the current dividend row and the counters, the worst case and typical case memory complexity is $O(1)$.

\subsubsection{Hash-Division for Divisor Groups}

In Section 3.3.6, we have analyzed optimizations of hash-division that require a dividend that is grouped on $Q$. We now show some optimizations of hash-division for a dividend that is grouped on $D$. Unlike the hash-division-like algorithms based on quotient groups, the following two algorithms are blocking.

This algorithm does not need a divisor hash table because after a divisor group of the dividend has been consumed, the divisor value will never reappear. We use a counter instead of a bitmap for each row in the quotient hash table. We call this adaptation of the HD algorithm hashdivision for divisor groups (HDD). The algorithm maintains a counter to count the number of divisor groups seen so far in the dividend. For each dividend row of a divisor group, we increment the counter of the quotient candidate. If the quotient candidate is not yet contained in the quotient hash table, we insert it together with a counter set to 1 . When the entire dividend has been processed, we return those quotient candidates in the quotient hash table whose counter is equal to the global counter.

\subsubsection{Transposed Hash-Division for Divi- sor Groups}

The last algorithmic adaptation that we present is called transposed hash-division for divisor groups (HDTD), based on the HDT algorithm. We can do without a divisor hash table, but we keep an array of counters during the scan of the dividend. The processing is basically the same as the previous algorithm (HDD): We return only those quotient candidates of the quotient hash table whose counter is equal to the value of the global counter. Because all divisor groups have to be processed before we know all quotients, this algorithm is also blocking.

We do not show the pseudo code for the HDD and HDTD algorithms for brevity. However, we sketch the data structures used in the Figures 6(c) and $(d)$ for the time when the entire dividend has been processed. Note that the dividend contains only three divisor groups (no Graphics rows), because we require that referential integrity between enrollment and course is preserved, e.g., by applying a semi-join of the two tables before division, as in Figure 4. Bob is the only student who is contained in all three divisor groups.

The complexities of HDD and HDTD are the same. Their worst and typical case time complexity is $O(|S|+|T|)$ and $O(|S|)$, respectively. The worst and typical case memory complexity is $O(|S|)$.

\subsubsection{Stream-Join Division}

The new algorithm stream-join division (SJD) [20] is an improvement of hash-division for divisor groups (HDD). As all other algorithms assuming a dividend that is grouped on $D$ as the only or the major set of group attributes, SJD is a blocking algorithm. SJD is hybrid because it counts the number divisor rows, like all other aggregate algorithms, and it maintains several bits to memorize matches between dividend and divisor, like all other scalar algorithms. However, in this paper, we consider SJD an aggregate algorithm due to its similarity to HDD.

The major differences between SJD and HDD are:

- SJD stores a bit instead of a counter together with each quotient candidate in the quotient hash table.

- SJD is able to remove quotient candidates from the quotient hash table before the end of the processing.

The SJD algorithm works as follows. As in HDD, we maintain a counter to count the number of divisor groups seen so far in the dividend. First, we insert all quotient candidates, i.e., $Q$ values, of the first group in the dividend together with a bit initialized with zero into the quotient hash table. We thereby eliminate possible duplicates in the dividend. Then, we process each following group as follows. For each dividend row of the current group, we look up the quotient candidate in the quotient hash-table. In case of a 
match, the corresponding bit is set to 1 . Otherwise, i.e., when the $Q$ value of a given dividend row is not present in the quotient hash table, we skip this row. After a group has been processed, we remove all quotient candidates with a bit equal to zero. Then, we reset the bit of each remaining quotient candidate to zero. Finally, when all groups have been processed, we compare the current group counter with the number of rows in the divisor. In case of equality, all quotient candidates in the quotient hash table with a bit equal to 1 are added to the output.

Figure 6(e) illustrates the use of the quotient hash table in SJD. We assume that the dividend is equal to the enrollment table of class 2 in Figure 2(b) with the exception that the Graphics group $\{($ Bob, Graphics), (Chris, Graphics) $\}$ is missing, due to referential integrity. We show the contents of the hash table for the time when the entire enrollment table has been processed. We see that Chris and Alice are not contained in the hash table because both have already been eliminated after the second group (Databases). Only Bob's bit is set to 1 and he is a quotient row because the number of groups (3, without Graphics) is equal to the number of divisor rows.

The advantage of SJD lies in the fact that the amount of memory can decrease but will never increase after the quotient candidates have been stored in the quotient hash table. However, the time and memory complexity is the same as for HDD. Observe that the maximum amount of memory required is proportional to the number of rows of the first group in the dividend. It may happen by chance that the first group is the smallest of the entire dividend. In this case, we obtain a very memory-efficient processing.

This algorithm is called stream-join division because it joins all divisor groups of the dividend (called streams in [20]) with each other on the attributes $Q$.

\section{Evaluation of Algorithms}

In this section, we briefly compare the division algorithms discussed in Section 3 with each other and show which algorithm is optimal, with respect to time and memory complexities, for each class of input data discussed in Section 2.

Table 3 characterizes the algorithms presented so far and shows the time and memory complexities involved. We assigned the algorithms to those data classes that have the least restrictions with respect to grouping. Remember that an algorithm of class $C$ can also process data of classes that are reachable from $C$ in the dependency graph in Figure 3. The overview of division algorithms in Table 3 shows that, despite the detailed classification in Table 1 (comprising 13 classes and enumerating all possible kinds of input data), there are four major classes of input data that are covered by dedicated division algorithms:

- class 0, which makes no assumption of grouping,

- class 2, which covers dividends that are grouped only or first on $D$,

- class 5, which covers dividends that are grouped only or first on $Q$, and finally

- class 10 , which specializes class 5 (and class 0 , of course) by requiring that for each quotient group, the rows of $D$ and the divisor appear in the same order. Hence, the dividend is grouped on $Q$ as major and $D$ as minor.

Note that algorithms for class 2, namely HDD, HDTD, and SJD, have not been identified in the literature so far. They represent a new straightforward approach to deal with a dividend that is grouped on $D$. Together with the other three major classes, a query optimizer can exploit the information on the input data properties to make an optimal choice of a specific division operator.

Suppose we are given input data of a class that is different from the four major classes. Which algorithms are applicable to process our data? According to the graph in Figure 3, all algorithms belonging to major classes, which are direct or indirect parent nodes of the given class, can be used. For example, any algorithm of major classes 0 and 5 can process data of the non-major classes 6,7 , and 9 . 


\begin{tabular}{|c|c|c|c|c|c|c|c|c|c|}
\hline \multirow{3}{*}{$\begin{array}{c}\text { Division } \\
\text { Algorithm }\end{array}$} & \multirow{3}{*}{$\begin{array}{l}\text { Algorithm } \\
\text { Class }\end{array}$} & \multirow{3}{*}{$\begin{array}{l}\text { Data } \\
\text { Class }\end{array}$} & \multirow{2}{*}{\multicolumn{2}{|c|}{ Dividend $S$}} & \multirow[t]{3}{*}{ Divisor $T$} & \multicolumn{4}{|c|}{ Complexity in $O$-Notation } \\
\hline & & & & & & \multicolumn{2}{|c|}{ Time } & \multicolumn{2}{|c|}{ Memory } \\
\hline & & & $\bar{Q}$ & $\bar{D}$ & & worst & typical & worst & typical \\
\hline$\overline{\mathrm{NLCD}}$ & aggregate & $\overline{00}$ & $\overline{N N}$ & $\overline{\bar{N}}$ & $\overline{\bar{N}}$ & $\overline{\mid \overline{|S|^{2}+\mid T}}$ & $\overline{\mid \overline{|S|^{2}}}$ & 1 & 1 \\
\hline NLD & scalar & & & & & $|S|^{2}+\mid T$ & $\mid \overline{|S|^{2}}$ & $|S|+|T|$ & $|S|$ \\
\hline HD & scalar & & & & & $|S|+|T|$ & $|S|$ & $|S||T|$ & $|S||T|$ \\
\hline HDT & scalar & & & & & $|S|+|T|$ & $|S|$ & $|S||T|$ & $|S||T|$ \\
\hline HDD & aggregate & 2 & $N$ & $G$ & $N$ & $|S|+|T|$ & $|S|$ & $|S|$ & $|S|$ \\
\hline HDTD & aggregate & & & & & $|S|+|T|$ & $\overline{|S|}$ & $|S|$ & $\overline{|S|}$ \\
\hline SJD & aggregate & & & & & $|S|+|T|$ & $|S|$ & $|S|$ & $|S|$ \\
\hline MCD & aggregate & 5 & $G$ & $\bar{N}$ & $\bar{N}$ & $|S|+|T|$ & $|S|$ & 1 & 1 \\
\hline HDQ & scalar & & & & & $|S|+|T|$ & $|S|$ & $|T|$ & 1 \\
\hline HDTQ & scalar & & & & & $|S|+|T|$ & $|S|$ & $|T|$ & 1 \\
\hline MGD & scalar & 10 & $G_{1}$ & $G_{2}$ & $G_{2}$ & $|S||T|$ & $|S||T|$ & $|T|$ & 1 \\
\hline MSD & scalar & & & $S_{2}$ & $S_{2}$ & $|S||T|$ & $|S||T|$ & 1 & 1 \\
\hline
\end{tabular}

Table 3

Overview of division algorithms showing for each algorithm the class of required input data, its algorithm class, and its time and memory complexities. Input data are either not grouped $(N)$, grouped $(G)$, or sorted $(S)$. Class 10 is first grouped on $Q$, indicated by $G_{1}$. For each quotient group, it is grouped $\left(G_{2}\right)$ or sorted $\left(S_{2}\right)$ on $D$ in the same order as the divisor. The algorithm names corresponding to the abbreviations in the first column are given in Table 2.

Several algorithms belong to each class of input data in Table 3. In class 0 , both HD and HDT have a linear time complexity (more precisely, nearly linear due to hash collisions). However, they have a higher memory complexity than the other algorithms of this class, NLCD and NLD.

We have designed three aggregate algorithms for class 2 . They all have the same linear time and memory complexities.

Class 5 has two scalar and one aggregate algorithm assigned to it, which all have the same time complexity. The constant worst case memory complexity of MCD is the lowest of the three.

The two scalar algorithms HDQ and HDTQ of class 10, which consists of two subgroups (sorted and grouped divisor values) have the same time complexity. The worst case memory complexity of MSD is lower than that of MGD because MSD can exploit the sort order.

It is important to observe that one should not directly compare complexities of scalar and aggregate algorithms in Table 3 to determine the most efficient algorithm overall. This is because aggregate algorithms require duplicate-free input tables, which can incur a very costly preprocess- ing step. There is one exception of aggregate algorithms: SJD ignores duplicate dividend rows because of the hash table used to store quotient candidates. It does not matter if a quotient occurs more than once inside a divisor group because the bit corresponding to a quotient candidate can be set to 1 any number of times without changing its value (1). However, SJD does not ignore duplicates in the divisor because it counts the number of divisor rows.

In general, scalar division algorithms ignore duplicates in the dividend and the divisor. Note that the scan operations of MGD and MSD can be implemented in such a way that they ignore duplicates in both inputs [8]. However, to simplify our presentation, the pseudo code of MSD in the Appendix does not ignore duplicates.

Let us briefly illustrate some example issues that we have to take into account when comparing division algorithms. The first issue is time versus memory complexity. In class 0 , for example, four algorithms have been identified. NLCD and NLD have a quadratic time complexity compared to the linear complexities of HD and HDT. Despite the different processing performance of 
these algorithms, a query optimizer may prefer to pick a division operator based on the NLCD algorithm to HD and HDT if the estimated amount of input data is small and the optimizer wants to avoid the overhead of building hash tables. We do not go into the details of query optimization here because, in general, the choice of picking a specific operator from a set of logically equivalent operators (like join and division) also depends on factors other than time and memory complexity, as we have mentioned in Section 2.2. Nevertheless, time and memory consumption are the dominant factors in reality.

The second issue is about the efficiency of a query processor for certain operations. We presented two different approaches for hash-division: the classic approach (HD), where bitmaps are stored together with quotient candidates in the quotient hash table, and a new approach (HDT) where bitmaps are stored with each divisor row in the divisor hash table (see Figures $5(\mathrm{~d})$ and (e) for illustrations). These dual approaches may seem interchangeable at first sight with respect to efficiency. However, in some situations, a query optimizer may prefer one to the other, depending on how efficiently the system processes bitmaps. Suppose the system can process a few extremely long bitmaps more efficiently than many short bitmaps. If there are many quotient candidates in the input data (which is typical) but there is a relatively short divisor, then the bitmaps stored in $\mathrm{HD}$ are relatively short but there are many of them. In contrast, HDT would build very long bitmaps (which may be the deciding factor) but only a few of them would be stored in the divisor hash table. Analogously, the optimizer may prefer HD to HDT if the input consists of few but very large quotient candidates. Similar situations apply to the other pairs of transposed and nontransposed algorithms, i.e., for the HDD/HDTD and HDQ/HDTQ pairs.

\section{The Set Containment Problem}

Universal quantification checks if all elements of a given set fulfill a given condition. In many applications, this condition is a set element membership test, i.e., the quantification problem becomes a set containment problem. For example, the problem stated in Section 1.1 can be rephrased as follows: "Find the students whose associated set of enrolled courses contains the given set of courses offered by the department."

\subsection{Set Storage Representations}

Division is an operator of the relational algebra, which is based on the relational model. In the basic relational model all relations are in first normal form (1NF), i.e., all attribute domains are atomic. One possible extension of the relational model provides relations with multivalued attributes, where the attribute domain is a collection type like bag or set, defined on top of a primitive domain like float or string. A more rigorous extension of the relational model is the nested relational model $[14,16]$, where attributes can be relations themselves.

There are basically two orthogonal classifications for the storage representation of sets: nesting and location [21]. The attribute values are stored as multiple values: the nested representation stores the values as a variable length attribute and the unnested representation stores them as multiple tuples.

In a classification based on the storage location, one can distinguish between an internal representation where the set elements are stored together with the accompanying attribute values and an external representation, where the set elements are stored in a separate auxiliary table connected by foreign key references, as depicted in Figure 7, according to [21]. In this figure, we show as an example a single tuple of the relation enrollment(student_id, courses), where student_id is an atomic attribute and courses is a set-valued attribute. Here, we represent the fact that the student Chris is enrolled in the courses Compilers, Graphics, and Theory. Only the unnested internal representation conforms to the $1 \mathrm{NF}$.

\subsection{Set Containment Join and Relational Division}

The set containment problem has been studied in great detail in the past $[10,17-19,21,22]$. In particular, several efficient set containment test algorithms have been developed and storage data 


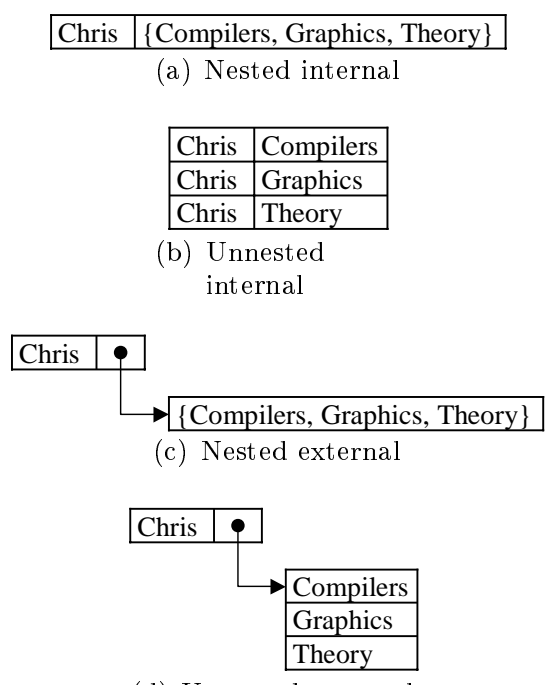

(d) Unnested external

Figure 7. Storage representations of set-valued attributes.

structures to represent sets in relational, objectrelational, and object-oriented databases are discussed.

It is interesting to observe that the division operator is closely related to set containment join, which can be implemented efficiently $[17,21]$. Set containment join (SCJ), denoted by $\bowtie_{\subseteq}$, is a join between the set-valued attributes $b$ and $\mathrm{c}$ of two relations $R(a, b)$ and $S(c, d)$ :

$$
S \bowtie_{c \subseteq b} R=\{t \mid t \in S \times R \wedge c \subseteq b\} .
$$

Figure 8 illustrates an example computation of the set containment join based on the scenario introduced in Section 1.1. Only the table course has been changed by adding an additional attribute program that indicates which combination of CS courses are required for a certain advanced program. We find that Bob has all prerequisites to specialize in Systems and Applications while Chris is only allowed to specialize in Applications.

Suppose, the tables course and enrollment are defined as before and that the layout of the setvalued attribute courses is unnested internal for both tables, as sketched in Figure 9. We have not

\begin{tabular}{|c|c|}
\hline \multicolumn{2}{|c|}{ enrollment } \\
\hline student_id & courses \\
\hline Alice & $\overline{\{\mathrm{C}, \mathrm{T}\}}$ \\
\hline Bob & $\{\mathrm{C}, \mathrm{D}, \mathrm{G}, \mathrm{T}\}$ \\
\hline Chris & $\{\mathrm{C}, \mathrm{G}, \mathrm{T}\}$ \\
\hline \multicolumn{2}{|c|}{ (a) $R(a, b)$} \\
\hline \multicolumn{2}{|c|}{ course } \\
\hline courses & program \\
\hline 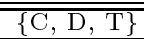 & Systems \\
\hline$\{\mathrm{C}, \mathrm{G}\}$ & Applications \\
\hline
\end{tabular}

(b) $S(c, d)$

course $\bowtie_{\text {courses } \subseteq \text { courses }}$ enrollment

\begin{tabular}{|l|l|l|l|}
\hline student_id & courses & courses & program \\
\hline \hline Bob & $\{\mathrm{C}, \mathrm{D}, \mathrm{G}, \mathrm{T}\}$ & $\{\mathrm{C}, \mathrm{D}, \mathrm{T}\}$ & Systems \\
\hline Bob & $\{\mathrm{C}, \mathrm{D}, \mathrm{G}, \mathrm{T}\}$ & $\{\mathrm{C}, \mathrm{G}\}$ & Applications \\
\hline Chris & $\{\mathrm{C}, \mathrm{G}, \mathrm{T}\}$ & $\{\mathrm{C}, \mathrm{G}\}$ & Applications \\
\hline \multicolumn{4}{|c}{ (c) $S \bowtie_{c} \subseteq b=T(a, b, c, d)$}
\end{tabular}

Figure 8. An example computation of the set containment join operator $\left(\bowtie_{\complement}\right)$ based on relations in non-first normal form employing a set-valued attribute.

\begin{tabular}{|c|c|c|c|}
\hline student_id & course_id & \multirow{2}{*}{\multicolumn{2}{|c|}{ course }} \\
\hline Alice & Compilers & & \\
\hline Alice & Theory & course_id & program \\
\hline Bob & Compilers & Compilers & Systems \\
\hline Bob & Databases & Databases & Systems \\
\hline Bob & Graphics & Theory & Systems \\
\hline Bob & Theory & Compilers & Applications \\
\hline Chris & Compilers & Graphics & Applications \\
\hline Chris & Graphics & \multirow{2}{*}{\multicolumn{2}{|c|}{ (b) $S(c, d)$}} \\
\hline Chris & Theory & & \\
\hline
\end{tabular}

(a) $R(a, b)$

\begin{tabular}{|c|c|}
\hline student_id & program \\
\hline$\overline{\mathrm{Bob}}$ & Systems \\
\hline Bob & Applications \\
\hline Chris & Applications \\
\hline
\end{tabular}

(c) $R \div b \supset c S=T(a, d)$

Figure 9. An example computation of the set containment division operator $(\div \supseteq)$ based on relations in first normal form.

found a definition of such a result table in a nested internal representation in the literature. Since all join attributes are preserved, it is unclear how 
the rows belonging to a set on the one side are combined with tuples of another set on the other side. One possible definition for representing the matches could be to pair each row from the left side with each row of the right side, i.e., one could compute the Cartesian product between the two groups of tuples that fulfill the set containment.

Because of this problem, we devised an extension of the division operator, called set containment division $(\div \supseteq)$ that returns the same rows as the set containment join but that delivers only the columns of the non-join attributes. Figure 9 illustrates the behavior of set containment division based on the same input data as in Figure 8 but using a $1 \mathrm{NF}$ data layout.

Formally, the set containment division can be expressed with the help of (basic) relational division as follows, again based on the two relations $R(a, b)$ and $S(c, d)$ :

$$
\begin{aligned}
T(a, d) & =R \div b \supseteq c \\
& =\bigcup_{x \in \pi_{d}(S)}\left(\left(R \div \pi_{c}\left(\sigma_{d=x}(S)\right)\right) \times(x)\right)
\end{aligned}
$$

The idea of this expression is to merge the result of several divisions. In each division, the entire dividend $R$ is divided by those tuples of the divisor $S$, which belong to the same group. There are as many divisions as the number of distinct values of S.d. We append the value of the current group to all result tuples of each division, specified by the Cartesian product.

We have seen that set containment join and relational division are very similar. We have demonstrated the similarity by defining an operator that operates on $1 \mathrm{NF}$ data like division but which can process many sets on both sides of the input like set containment join. The characteristics of the three operators discussed before are summarized in Table 4.

\subsection{Overview of Set Containment Join Al- gorithms}

The set containment join algorithms that have been proposed in the literature are based on signatures [10] and partitioning [22]. Enhanced approaches combining both techniques have been developed, which significantly outperform all known previous approaches [17-19]. All these al- gorithms assume that the data is managed by the database system in a non-1NF way, i.e., the data can be everything but unnested internal, which is the layout assumed for the set containment division and basic division problems. However, in [19] the new approaches are compared to SQLbased approaches based on counting the number of elements in the join result of both sets and comparing it to the set cardinality of the candidate subset. Such a comparison is incomplete because other SQL-based approaches using NOT EXISTS (as for division) have not been taken into consideration, as described in Section 3.2.

A recent study compared set containment joins based on a nested internal and an unnested internal set representation [11], also based on the counting approach, only. In particular, in the nested approach, a user-defined containment test predicate is employed that takes two set-valued attributes as parameters. According to current database technology for evaluating user-defined predicates, the commercial system in use is forced to apply the test predicate on the result table of a Cartesian product of both input tables. By rewriting the query into one using an unnested layout, a table function is employed that unnests the set-valued attribute into a table. The optimizer of the system used in their experiments decided to first build an intermediate result table that comprises the set ID and the element value as attributes, sorted on the element values. Then, the query execution plan suggests to merge-join the two sorted input streams on the element value attributes. After that, the sorted data is grouped on the set IDs and set cardinalities. Finally, a filter condition appends only those set ID pairs to the result, where the cardinality of the contained set is equal to the number of matches for this pair of sets. The experiments of this study have shown that the effort of unnesting the sets and preprocessing the data by sorting it on the attributes to be matched can greatly improve the straightforward nested-loops approach. Unfortunately, the results have not been compared to more sophisticated approaches as the ones proposed, for example, in [17].

The research results on relational division should be applied to set containment in future 


\begin{tabular}{|l|l|l|l|}
\cline { 2 - 4 } \multicolumn{1}{c|}{} & Division & Set Containment Division & Set Containment Join \\
\hline Operator and input relations & $R(a, b) \div S(c)$ & $R(a, b) \div{ }_{b \supseteq c} R(c, d)$ & $S(c, d) \bowtie_{c} \subseteq b R(a, b)$ \\
\hline Left input / dividend & many groups & many groups & many sets \\
\hline Right input / divisor & single group & many groups & many sets \\
\hline Result / quotient attributes & $T(a)$ & $T(a, d)$ & $T(a, b, c, d)$ \\
\hline Data layout & $1 \mathrm{NF}$ & $1 \mathrm{NF}$ & non-1NF \\
\hline
\end{tabular}

Table 4

Summary of operator characteristics

work since the division problem can be considered a sub-problem of set containment join under the assumption that the sets are stored using an unnested internal representation. The main difference between both operations is that division is applied to a single set of dividend set elements, whereas set containment join compares possibly multiple sets from both sides of the join with each other. To the best of our knowledge, the strong commonality between relational division and set containment join has not been identified and investigated before.

\section{New Applications for Universal Quan- tification}

In this section, we first argue why business intelligence problems are likely to benefit from being expressed using SQL. Then, we suggest a novel approach to compute frequent itemsets - a popular data mining task-which employs universal quantifications in the SQL queries.

\subsection{Database Mining}

In business intelligence applications, several data mining and OLAP techniques are employed to extract novel and useful information from huge corporate data sets. Typically, the data sets are managed by a data warehouse that is based on relational database technology. Although the term data mining and, even more so, knowledge discovery in databases (KDD) suggest that the algorithms explore databases, most commercial tools merely process flat files. If they do access a database system, then database tables are used as a container to read and write data, similar to a file system. The query optimization and processing facilities of current database systems are hardly ever exploited by current data mining tools. The reasons for this certainly include:

- Portability: A data mining application that does not rely on a query language can be deployed more easily because no assumptions on the language's functionality have to be made.

- Performance: A highly tuned black-box algorithm with in-memory data structures will always be able to outperform any query processor that employs a combination of generic algorithms.

- Secrecy: A tool vendor does not want to reveal application logic. By employing SQLbased algorithms, the database administrator will be able to see these queries.

Despite these arguments against SQL-based data mining algorithms, exploiting the query language power for expressing data mining (sub)problems can solve several important problems:

- Data currency: The latest updates applied to the data warehouse are reflected in the query result. No (replicated) data copies have to be maintained.

- Scalability: If extremely large data sets are to be mined then it is much easier to design a scalable SQL-based algorithm than designing an algorithm that has to manage data in external files. The storage management is one of the key strengths of a database system.

- Adaptability to data: A database optimizer tries to find the best possible execution 
strategy based on the current data characteristics for a given query. Of course, in some situations this will not help. Similar to choosing a different proprietary algorithm for certain data characteristics, it may be better to employ a different query.

The latter three arguments motivated our research on SQL-based algorithms for several data mining methods. One of these methods is discussed in the following section.

\subsection{Frequent Itemset Discovery with SQL}

In this section, we first briefly introduce the frequent itemset discovery problem and explain the relationship between frequent itemset discovery and relational division and set containment join. Then, we present a new approach for this problem, which makes use of universal quantifications.

\subsubsection{The Frequent Itemset Discovery Problem}

The computation of frequent itemsets is a computationally expensive preprocessing step for association rule discovery, which finds rules in large transactional data sets [1]. Frequent itemsets are combinations of items that appear frequently together in a given set of transactions. Association rules characterize, for example, the purchasing pattern of retail customers or the click pattern of web site visitors. Such information can be used to improve marketing campaigns, retailer store layouts, or the design of a web site's contents and hyperlink structure.

Given a set of transactions, the frequent itemset discovery problem is to find itemsets within the transactions that appear at least as frequently as a given threshold, called minimum support. For example, a user can define that an itemset is frequent if it appears in at least $2 \%$ of all transactions.

Almost all frequent itemset discovery algorithms consist of a sequence of steps that proceed in a bottom-up manner: the result of the $k$-th step is the set of frequent $k$-itemsets, denoted as $F_{k}$. The first step computes the set of frequent items (1-itemsets). Each following step consists of two phases:
1. The candidate generation phase computes a set of potentially frequent $k$-itemsets from $F_{k-1}$. The new set is called $C_{k}$, the set of candidate $k$-itemsets. It is a superset of $F_{k}$.

2. The support counting phase filters out those itemsets from $C_{k}$ that appear more frequently in the given set of transactions than the minimum support and stores them in $F_{k}$.

The key problem of frequent itemset discovery is: "How many transactions contain a certain given itemset?" This question can be answered in relational algebra using the division operator. Suppose that we have a relation Transaction(tid, item) containing a set of transactions and a relation Itemset(item) containing a single itemset, each row containing one item. We want to collect those tid values in a relation Contains (tid), where for all tuples in Itemset there is a corresponding tuple in Transaction that has a matching item value together with that $t i d$. In relational algebra, this problem can be stated as

$$
\begin{aligned}
& \text { Transaction }(\text { tid, item }) \div \text { Itemset }(\text { item }) \\
= & \text { Contains }(\text { tid }) .
\end{aligned}
$$

The example in Figure 10 illustrates the division operation. The Transaction table consists of three transactions and two of them contain all items of Itemset. We simply have to count the values in Contains to decide if the itemset is frequent. For example, if the minimum support is set to $60 \%$ then the given itemset is considered a frequent itemset because the support is $2 / 3$, which is greater than $60 \%$. Using division terminology, Transaction plays the role of the dividend, Itemset represents the divisor, and Contains is the quotient.

Unfortunately, frequent itemset discovery poses the additional problem that we have to check many (candidate) itemsets if they are frequent, i.e., unlike Figure 10(b), we usually do not have a constant divisor relation but we need many divisor relations. However, we can employ efficient algorithms for this problem. We could arrange the itemsets in a table Itemset (itemset, item) 
Transaction

\begin{tabular}{|l|l|}
\hline tid & item \\
\hline \hline 1001 & diapers \\
\hline 1001 & beer \\
\hline 1001 & chips \\
\hline 1002 & chips \\
\hline 1002 & diapers \\
\hline 1003 & beer \\
\hline 1003 & avocados \\
\hline 1003 & chips \\
\hline 1003 & diapers \\
\hline
\end{tabular}

(a) Dividend

\begin{tabular}{|l|l|}
\multicolumn{1}{l}{ Itemset } & Contains \\
\hline item & tid \\
\hline \hline chips & \\
\hline \hline beer & 1001 \\
\hline diapers & 1003 \\
\hline (b) Divisor & (c) Quotient
\end{tabular}

(b) Divisor

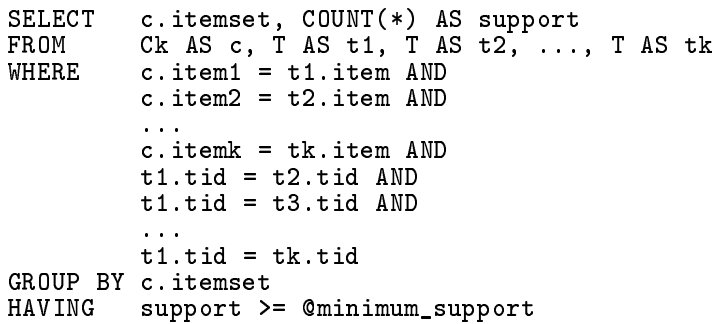

Figure 11. Support counting phase according to the K-Way-Join algorithm.

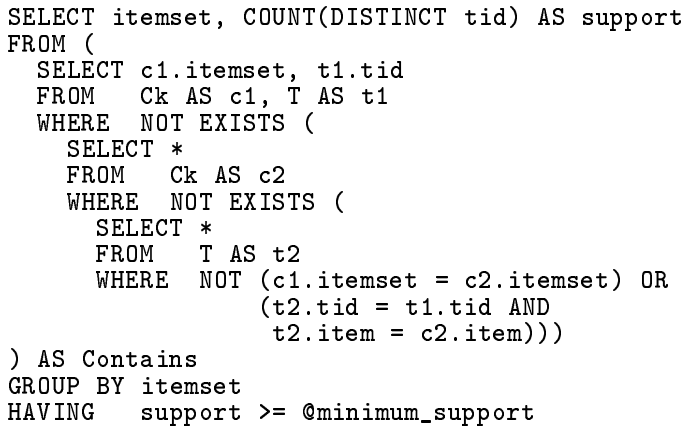

$1 \mathrm{NF}$ tables would have a schema like: Itemset (itemset, itemvalues) and Transaction (tid, itemvalues), each having a set-valued attribute itemvalues.

\subsubsection{Support Counting in SQL}

In this paper, we focus on the support counting phase of frequent itemset discovery. For typical data sets, this phase is much more computationally expensive than the candidate generation phase. 
There are several approaches to express the support counting phase in SQL. Most of them are based on SQL-92. The SETM algorithm is the first SQL-based approach described in the literature [12]. Several researchers have suggested improvements of SETM. It has been shown that SETM does not perform well on large data sets and new approaches have been devised, like for example Three-Way-Join, Subquery, and TwoGroup-Bys [25]. The algorithms presented in that paper perform differently for different data characteristics. Subquery is reported to be the best approach overall compared to the other approaches based on SQL-92. The reason is that it exploits common prefixes between candidate $k$ itemsets when counting the support.

More recently, an approach called Set-Oriented Apriori has been proposed [27]. The authors argue that too much redundant computations are involved in each support counting phase. Their performance results have shown that SetOriented Apriori performs better than Subquery, especially for high values of $k$.

We contrast our novel approach to previous approaches based on SQL-92 where the data is stored in $1 \mathrm{NF}$, i.e., we do not investigate setvalued attributes, for example. One of the approaches based on SQL-92 is K-Way-Join [25], illustrated in Figure 11. The K-Way-Join approach, which is based on SQL-92, uses $k$ instances of the transaction table and joins it $k$ times with itself and with a single instance of $C_{k}$. Same as all other known approaches based on SQL-92 that use a 1NF representation of itemsets, K-Way-Join assumes that the frequent and candidate $k$-itemsets are stored in a single row: (itemset, item $_{1}, \ldots$, item $m_{k}$ ). However, the given transactions are stored as multiple rows using the schema (tid, item). As we will show in the following section, our novel approach uses a data layout where itemsets are stored as multiple rows, same as the transactions.

\subsubsection{Support Counting and Universal Quantification}

Based on the idea of using division to specify the itemset containment problem, we devised a complete algorithm, called Quiver (QUantified
Itemset discovery using a VERtical table layout) [23], that employs SQL queries containing universal quantifications for both phases of the discovery task. The reason for devising a new approach is twofold:

1. We want to formulate intuitive queries that naturally express the universal quantification problem: "Count the number of transactions where for each transaction, all items of a given itemset are contained in the transaction." Previous approaches for SQLbased frequent itemset discovery are mostly "hardwired" queries, i.e., the quantification is circumvented by using many join conditions between individual items of candidates and transactions (as shown for the $\mathrm{K}$ Way-Join approach in the previous section).

2. We want to employ a flexible itemset representation that is similar to the way transactions are stored in a database: Transaction (tid,item). In all previous approaches that use a $1 \mathrm{NF}$ representation, $k$-itemsets are stored as a single row: (itemset, item $_{1}, \ldots$, item it $_{k}$ ). Instead of this "horizontal" layout, Quiver uses a "vertical" layout, where a $k$-itemset is represented as $k$ rows in the three-column table (itemset, position, item). One benefit of this vertical layout is its ability to store even very large itemsets because in commercial database systems the maximum number of columns in a table is significantly lower than the number of rows.

In the following, we describe only the support counting phase of Quiver and we focus on the core problem, universal quantification. The entire approach, including the candidate generation phase using universal quantification, is described in detail in [23].

The query for support counting is first presented with help of tuple relational calculus since the calculus offers a universal quantifier to conveniently express the quantification. After this, we show how to derive an equivalent SQL query. As explained in Section 3.2, SQL does not offer a universal quantifier, therefore the query is expressed 
with the help of negated existential quantifiers.

Since Quiver follows the classical iterative two-phase approach, suppose that we have computed the set of candidate $k$-itemsets $C_{k}$ (itemset, position, item) based on the set of frequent $(k-1)$-itemsets $F_{k}$ (itemset, position, item) during the first phase of the $k$-th iteration, with $k \geq 2$. The set of transactions is given by table $T$ (tid, item).

We express the query $Q$ in tuple relational calculus to derive combinations of transactions and candidates as

$Q=\left\{\left(c_{1} \in C, t_{1} \in T\right) \mid\right.$ Contains $\}$.

The query can be applied to candidate itemsets of any size. Therefore, the parameter $k$ of the particular candidate set $C_{k}$ is omitted for brevity. The Contains expression of this query is defined as

$$
\begin{aligned}
\text { Contains }= & \forall c_{2} \in C \exists t_{2} \in T \\
& \left(c_{2} . \text { itemset }=c_{1} . \text { itemset }\right) \rightarrow \\
& \left(t_{2} . \text { tid }=t_{1} . \text { tid } \wedge\right. \\
& \left.t_{2} . \text { item }=c_{2} . \text { item }\right) .
\end{aligned}
$$

The expression has two free tuple variables $c_{1}$ and $t_{1}$, where $c_{1}$ represents a candidate itemset and $t_{1}$ is a transaction that contains $c_{1}$. The quantified (bound) tuple variables $c_{2}$ and $t_{2}$ represent the items belonging to $c_{1}$ and $t_{1}$, respectively. The universal quantification lies in the condition that for each item $c_{2}$ belonging to itemset $c_{1}$, there must be an item $t_{2}$ belonging to transaction $t_{1}$ that matches with $c_{2}$.

A combination $\left(c_{1}, t_{1}\right)$ fulfilling the calculus query $Q$ indicates that the itemset $c_{1}$.itemset is contained in the transaction $t_{1}$.tid. We can find the support of each candidate by counting the number of distinct values $t_{1}$.tid that appear in a combination $c_{1}$.itemset. We do not show the actual counting because the basic calculus does not include aggregate functions.

Since we are interested in an SQL representation of the given calculus query, we translate it into SQL in a straightforward manner by applying the following transformations:
- Quantifiers: As already explained before, there is no universal quantifier available in SQL. Therefore, we translate $\forall x \in R: f(x) \equiv \neg \exists x \in R$ : $f(x)$ into "NOT EXISTS (SELECT * FROM R AS $x$ WHERE NOT $f(x))$."

- Implications: We replace an implication by a disjunction, i.e., we transform $f \rightarrow g \equiv$ $\neg f \vee g$ into "NOT f OR g."

- Negations: We use De Morgan's rules $\neg(f \wedge$ $g) \equiv \neg f \vee \neg g$ and $\neg(f \vee g) \equiv \neg f \wedge \neg g$ for pushing a negation into a conjunction or a disjunction.

The resulting SQL query for support counting, shown in Figure 12, contains two nested "NOT EXISTS" expressions analogous to the example SQL query used to express the student's enrollment problem in Section 3.2. Note that the query in Figure 12 has to apply the aggregation on the set of unique transaction IDs because duplicates can occur as a result of the query processing.

To conclude this section, we point out that the Quiver approach shows how an important data mining task can be expressed in a natural way using universal quantification. If a database system were able to recognize the quantification problem inside queries like the one in Figure 12, it could employ the most efficient algorithm that realizes the division operator, set containment division operator, or set containment join operator (discussed in Section 5), taking into account the current data characteristics, as explained in the previous sections. This is especially important if the data mining problem is a part of a larger, more complex query, involving several additional predicates. For example, consider a supermarket scenario, where we restrict our analysis to transactions of the years 1999-2001, and we are only interested in items of the product category "soft drinks." Such additional predicates can significantly influence the choice on the most efficient algorithm for the quantification problem.

\section{Related Work}

Quantifiers in queries can be expressed by relational algebra. Due to the lack of efficient di- 
vision algorithms in the past, early work has recommended avoiding the relational division operator to express universal quantification in queries [3]. Instead, universal quantification is expressed with the help of the well-known anti-semi-join operator, or complement-join, as it is called in that paper.

Other early work suggests approaches other than division to process (universal) quantification $[5,6]$. Universal quantification is expressed by new algebra operators and is optimized based on query graphs in a non-relational data model [6]. Due to the lack of a performance analysis, we cannot comment on the efficiency of this approach.

The research literature provides only few surveys of division algorithms $[4,7,8]$. Some of the algorithms reviewed in this paper have been compared both analytically and experimentally [8]. The conclusion is that hash-division outperforms all other approaches. Complementing this work, we have shown that an optimizer has to take the input data characteristics and the set of given algorithms into account to pick the best division algorithm. The classification of four division algorithms in [8] is based on a two-by-two matrix. One axis of the matrix distinguishes between algorithms based on sorting or based on hashing. The other axis separates "direct" algorithms, which allow processing the (larger) dividend table only once, from "indirect" algorithms, which require duplicate removal (by employing semi-join) and aggregation. For example, the merge-sort division algorithm of Section 3.3.2 falls into the category "direct algorithm based on sorting," while the hash-division for divisor groups algorithm of Section 3.4.3 belongs to the combination "indirect algorithm based on hashing." Our classification details these four approaches and focuses on the fact that data properties should be exploited as much as possible by employing "slim" algorithms that are separated from preprocessing algorithms, like grouping and sorting.

Based on a classification of queries that contain universal quantification, several query evaluation techniques have been analyzed [4]. The input data of this algorithm analysis is stored in an object-oriented or object-relational database, where set-valued attributes are available. Hence, the algorithms they examine can presuppose that the input data is grouped on certain attributes. For example, the table enrollment in Figure 1 could be represented by a set-valued $e n$ rolled_courses attribute of a student class. The authors conclude that universal quantification based on anti-semi-join is superior to all other approaches, similar to the conclusion of [3]. Note, however, that this paper has a broader definition of queries involving universal quantification than the classic definition that involves the division operator. However, the anti-semi-join approach requires a considerable overhead for preprocessing the dividend. An equivalent definition of the division operator using anti-semi-join $(\bar{\ltimes})$ as well as semi-join $(\ltimes)$ and left outer join $\left(\aleph_{l o}\right)$, is: $S \div T=\left((S \ltimes T) \bowtie_{l o} T\right) \bar{\ltimes} T$.

In this paper, we focused on the universal (forall) quantifier. Generalized quantifiers have been proposed to specify quantifiers like "at least ten" or "exactly as many" in SQL [13]. Such quantifiers can be processed by algorithms that employ multi-dimensional matrix data structures [24]. In that paper, however, the implementation of an operator called all is presented that is similar but different from relational division. Unlike division, the result of the all operator contains some attributes of the divisor. Hence, we have to employ a projection on the quotient attributes of the all operator's result to achieve a valid quotient.

Transformation rules for optimizing queries containing multiple (existential and universal) quantifications are presented in [15]. Our contribution complements this work by offering strategies to choose a single (division) operator, which may be one element of a larger query processing problem.

\section{Conclusion and Future Work}

Based on a classification of input data properties, we were able to differentiate the major currently known algorithms for relational division. In addition, we could provide new algorithms for previously not supported data properties. Thus, for the first time, an optimizer has a full range of algorithms, separated by their input data proper- 
ties and efficiency measures, to choose from.

We are aware of the fact that database system vendors are reluctant to implement several alternative algorithms for the same query operator, in our case the division operation. One reason is that the optimizer's rule set has to be extended, which can lead to a larger search space for queries containing division. Another reason is that the optimizer must be able to detect a division in a query. This is a non-trivial task because a division cannot be expressed in SQL:1999 [2]. No keyword similar to "FOR ALL" [9] is available and division has to be expressed indirectly, for example by using nested "NOT EXISTS" clauses or by using the "division by counting" approach on the query language level. To the best of our knowledge, there is no database system that has an implementation of hash-division (or any of its improvements), although this efficient algorithm has been known for many years [7]. However, we believe that as soon as a dedicated keyword for universal quantification is supported by the SQL standard and its benefit is recognized and exploited by applications, many options and strategies are available today for database system vendors to implement an efficient division operator.

The similarity between relational division and the set containment join has been discussed for the first time. This may lead to more research that investigates the possibility of representing sets in an unnested storage layout because efficient algorithms for division can be exploited. We have proposed a new operator, called set containment division, that realizes set containment joins for data in first normal form.

We have discussed an important application of the division (and hence set containment) problem, namely frequent itemset discovery. We plan to investigate the potential of using universal quantification in queries in further data mining methods of business intelligence applications.

Our future work includes the analysis of further data properties that have an influence on the optimization of division queries, like the current data distribution or the availability of certain indexes. Furthermore, we will study the potential of parallelizing division algorithms, based on the detailed studies in [8] on parallelizing hash- division and aggregate algorithms. In addition, the comparison between division and set containment join algorithms deserves more attention. In particular, further investigations of both operators need to take into account the cost of nesting and unnesting between the $1 \mathrm{NF}$ and the non-1NF storage representations of sets in order to provide fair performance comparisons.

\section{REFERENCES}

1. R. Agrawal and R. Srikant. Fast Algorithms for Mining Association Rules. In Proceedings VLDB, Santiago, Chile, pages 487-499, September 1994.

2. ANSI/ISO/IEC 9075-2. Information Technology - Database Language - SQL - Part 2: Foundation (SQL/Foundation), 1999.

3. F. Bry. Towards an Efficient Evaluation of General Queries: Quantifier and Disjunction Processing Revisited. In Proceedings SIGMOD, Portland, Oregon, USA, pages 193204, May-June 1989.

4. J. Claußen, A. Kemper, G. Moerkotte, and K. Peithner. Optimizing Queries with Universal Quantification in Object-Oriented and Object-Relational Databases. In Proceedings VLDB, Athens, Greece, pages 286-295, August 1997.

5. U. Dayal. Queries with Quantifiers: A Horticultural Approach. In Proceedings PODS, Atlanta, Georgia, USA, pages 125-136, March 1983.

6. U. Dayal. Of Nests and Trees: A Unified Approach to Processing Queries that Contain Nested Subqueries, Aggregates, and Quantifiers. In Proceedings VLDB, Brighton, England, pages 197-208, September 1987.

7. G. Graefe. Query Evaluation Techniques for Large Databases. TKDE, 25(2):73-170, June 1993.

8. G. Graefe and R. Cole. Fast Algorithms for Universal Quantification in Large Databases. TODS, 20(2):187-236, 1995.

9. P. Gulutzan and T. Pelzer. SQL-99 Complete, Really: An Example-Based Reference Manual of the New Standard. R\&D Books, Lawrence, Kansas, USA, 1999. 
10. S. Helmer and G. Moerkotte. Evaluation of Main Memory Join Algorithms for Joins with Set Comparison Join Predicates. In Proceedings VLDB, Athens, Greece, pages 386-395, August 1997.

11. S. Helmer and G. Moerkotte. Compiling Away Set Containment and Intersection Joins. In Technical Report 04/02, University of Mannheim, Germany, April 2002.

12. M. Houtsma and A. Swami. Set-Oriented Data Mining in Relational Databases. DKE, 17(3):245-262, December 1995.

13. P. Hsu and D. Parker. Improving SQL with Generalized Quantifiers. In Proceedings ICDE, Taipei, Taiwan, pages 298-305, March 1995.

14. G. Jaeschke and H.-J. Schek. Remarks on the Algebra of Non First Normal Form Relations. In Proceedings PODS, Los Angeles, California, USA, pages 124-138, March 1982.

15. M. Jarke and J. Koch. Range Nesting: A Fast Method to Evaluate Quantified Queries. In Proceedings SIGMOD, San Jose, California, USA, pages 196-206, May 1983.

16. A. Makinouchi. A Consideration on Normal Form of Not-Necessarily-Normalized Relation in the Relational Data Model. In Proceedings VLDB, Tokyo, Japan, pages 447-453, October 1977.

17. S. Melnik and H. Garcia-Molina. Adaptive Algorithms for Set Containment Joins. Technical Report, Department of Computer Science, Stanford University, California, USA, November 2001.

18. S. Melnik and H. Garcia-Molina. Divideand-Conquer Algorithm for Computing Set Containment Joins. In Proceedings EDBT, Prague, Czech Republic, pages 427-444, March 2002.

19. S. Melnik and H. Garcia-Molina. Divide-andConquer Algorithm for Computing Set Containment Joins. Extended Technical Report, Stanford University, California, USA, 2002.

20. C. Nippl, R. Rantzau, and B. Mitschang. StreamJoin: A Generic Database Approach to Support the Class of Stream-Oriented Applications. In Proceedings IDEAS, Yokohama, Japan, pages 83-91, September 2000.
21. K. Ramasamy. Efficient Storage and Query Processing of Set-Valued Attributes. PhD thesis, University of Wisconsin, Madison, Wisconsin, USA, 2002. 144 pages.

22. K. Ramasamy, J. Patel, J. Naughton, and R. Kaushik. Set Containment Joins: The Good, The Bad and The Ugly. In Proceedings VLDB, Cairo, Egypt, pages 351-362, September 2000.

23. R. Rantzau. Frequent Itemset Discovery with SQL Using Universal Quantification. In Proceedings Workshop on Database Technology for Data Mining (DTDM), Prague, Czech Republic, pages 51-66, March 2002.

24. S. Rao, A. Badia, and D. v. Gucht. Providing Better Support for a Class of Decision Support Queries. In Proceedings SIGMOD, Montreal, Canada, pages 217-227, June 1996.

25. S. Sarawagi, S. Thomas, and R. Agrawal. Integrating Association Rule Mining with Relational Database Systems: Alternatives and Implications. In Proceedings SIGMOD, Seattle, Washington, USA, pages 343-354, June 1998.

26. J. Smith and P. Chang. Optimizing the Performance of a Relational Algebra Data Base Interface. $C A C M, 18(10): 568-579$, October 1975.

27. S. Thomas and S. Chakravarthy. Performance Evaluation and Optimization of Join Queries for Association Rule Mining. In Proceedings DaWaK, Florence, Italy, pages 241250, August-September 1999.

28. W3C. XQuery 1.0: An XML Query Language. Working Draft 7, W3C, 2001.

\section{Appendix: Pseudo Code of Division Algo- rithms}

The following algorithms in Figures 13-17 assume that the division's input consists of a dividend table $S$ (quotient, divisor) and a divisor table $T$ (divisor). Furthermore, we use the variables $s$ and $t$ to refer to a single row within $S$ and $T$, respectively. The data structures $d h t$ and $q h t$ represent a divisor hash table and a quotient hash table, respectively. 


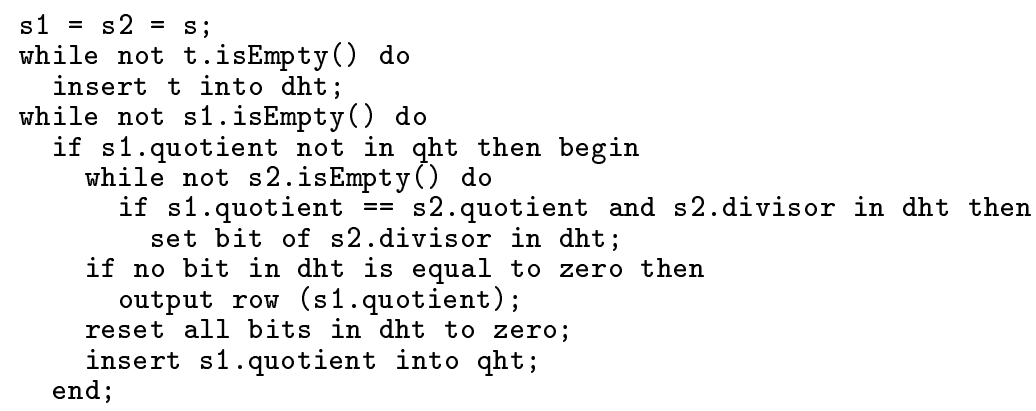

Figure 13. Nested-Loops Division (Class 0)

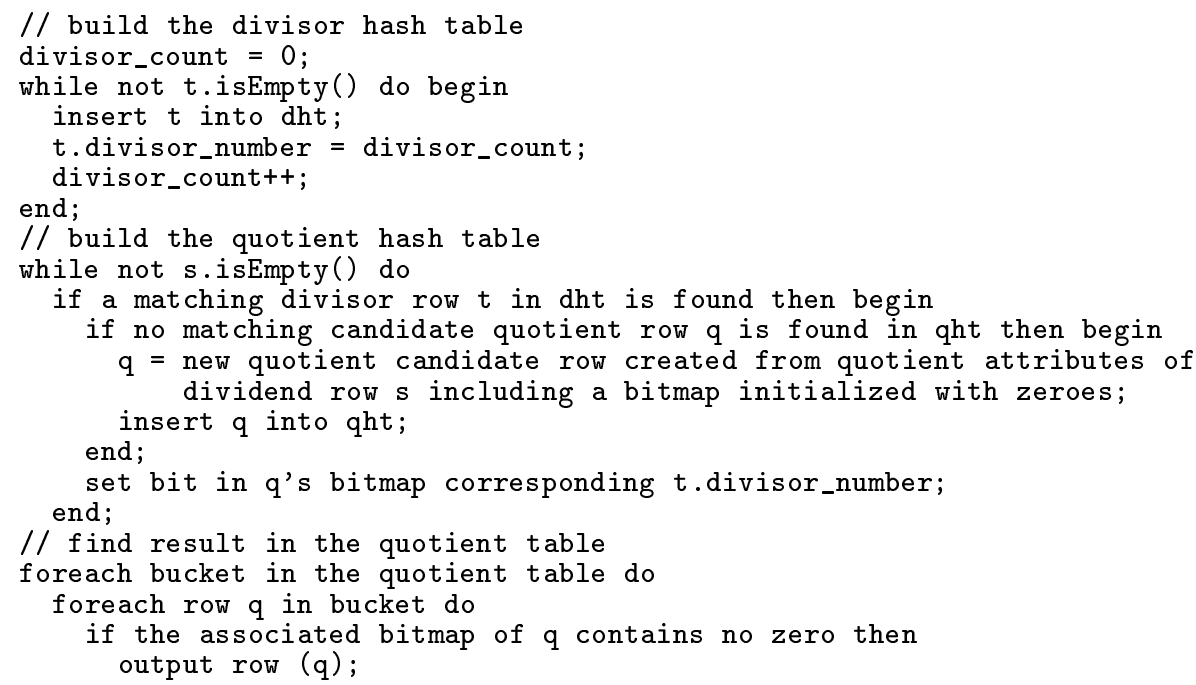

Figure 14. Classic Hash-Division (Class 0) 


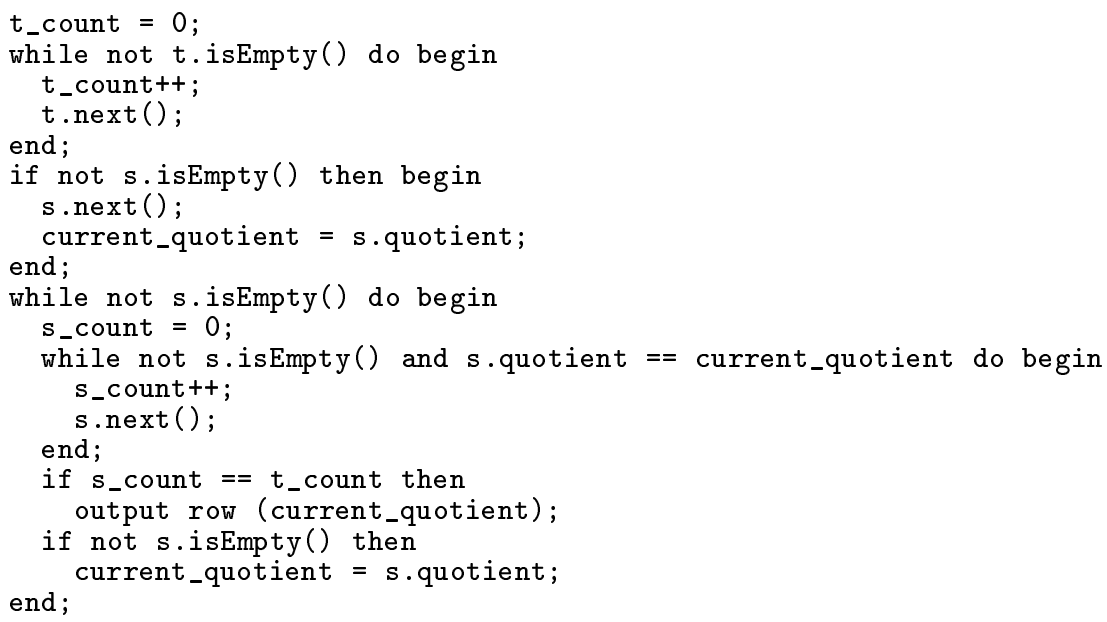

Figure 15. Merge-Count Division (Class 5)

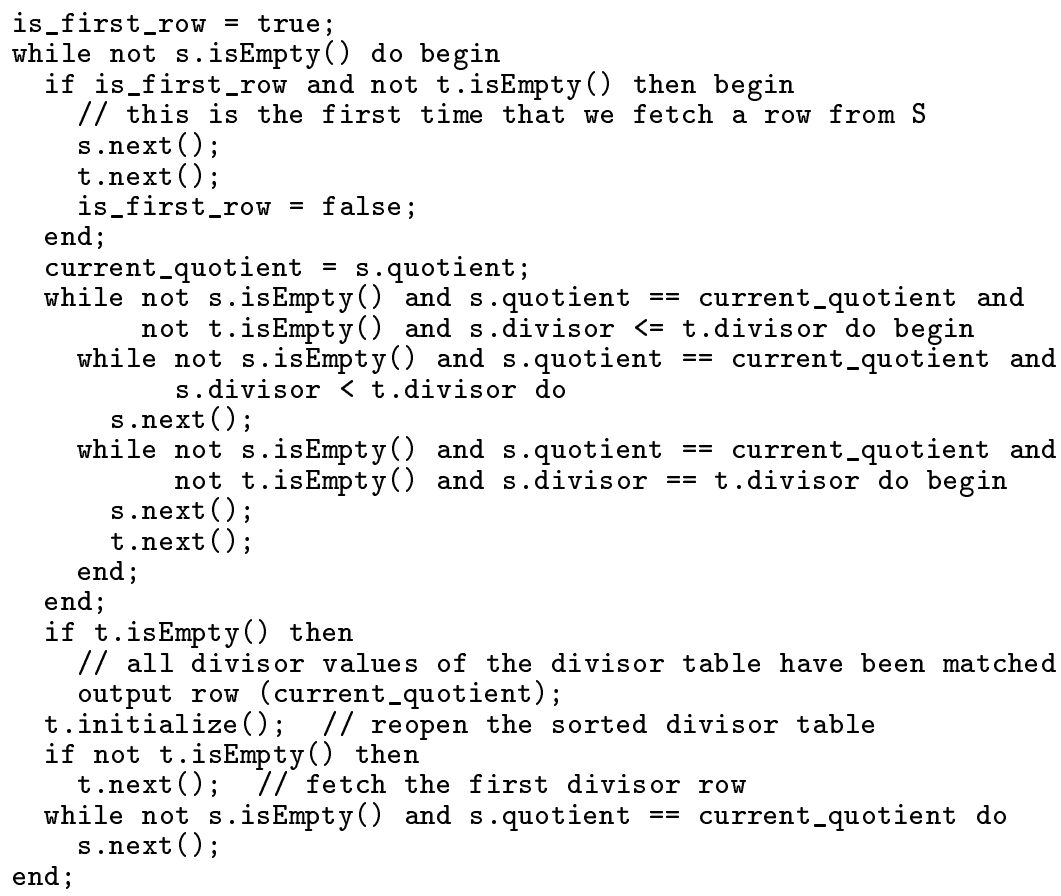

Figure 16. Merge-Sort Division (Class 10). Without loss of generality, the pseudo code assumes an ascending sort order. 


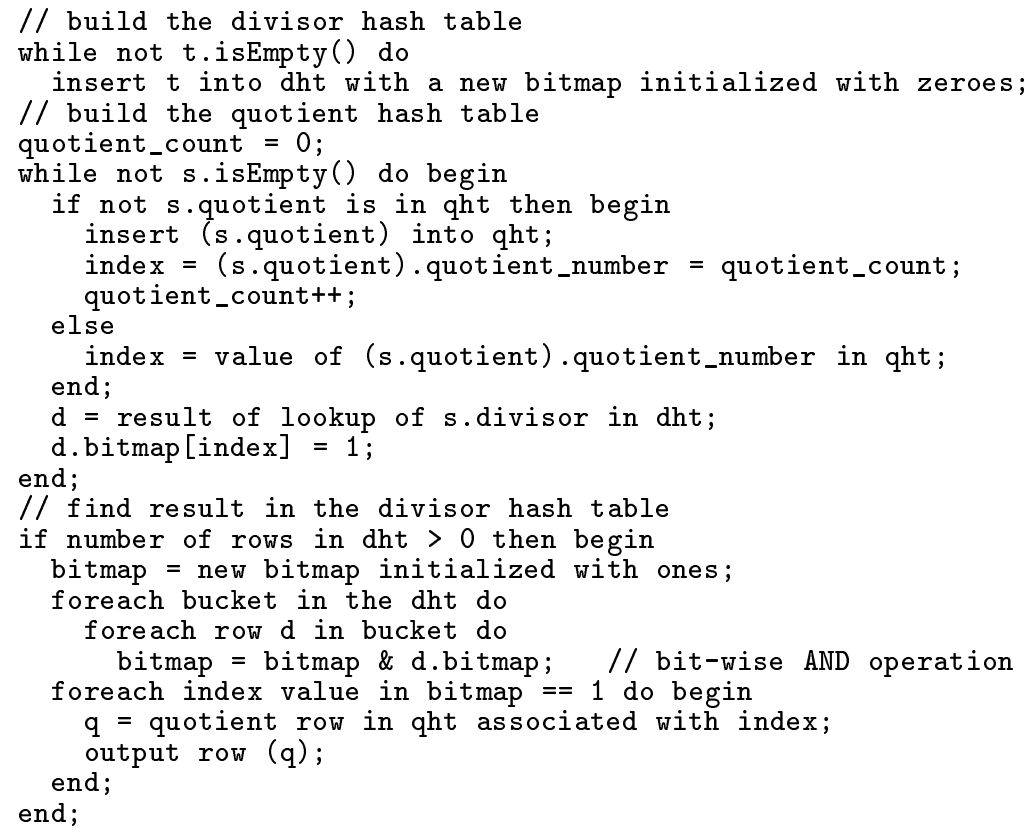

Figure 17. Transposed Hash-Division (Class 0) 